\title{
Le Langage des Espaces et des Groupes Quantiques
}

\author{
Georges Maltsiniotis \\ UFR de Mathématiques, Université de Paris VII, Tours 45-55 5ème étage, 2, Place Jussieu, \\ F-75251 Paris Cedex 05, France
}

Received March 3, 1992; in revised form June 5, 1992

\begin{abstract}
We study the foundations of the differential calculus in quantum geometry. The notions of (differential) quantum space and cone are introduced. Generalizing a construction of Manin, to a quantum cone we associate the quantum group of its "linear automorphisms preserving the differentials" and deduce a de Rham complex on this group. We give examples of differential calculi on quantum hyperplanes and quantum linear groups.
\end{abstract}

\section{Introduction}

Le but de cet article est de présenter une étude systématique d'un calcul différentiel non commutatif, dans le cadre des espaces et des groupes quantiques. Dans l'esprit du livre de Yu. I. Manin, "Quantum groups and non-commutative geometry," un espace quantique est une "variété algébrique" en "géométrie algébrique non commutative." Par analogie à la géométrie commutative ordinaire, on est tenté d'identifier les " $K$ espaces quantiques affines," où $K$ désigne un corps commutatif (ou plus généralement un anneau commutatif), aux objets de la catégorie opposée à celle des $K$-algèbres associatives, unifères, non nécessairement commutatives. Néanmoins, si en géométrie algébrique commutative, la donnée de l'anneau structural d'un schéma affine définit canoniquement une "structure différentielle" sur ce schéma, il n'en est pas de même dans le cas non commutatif. Plus précisement, si $A$ est une $K$-algèbre commutative, et si l'on désigne par $\mu: A \otimes A \rightarrow A$ l'application $K$-linéaire définissant la multiplication de $A$, le noyau $I$ de $\mu$ est un idéal de $A \otimes A$, l'application $K$-linéaire $d: A \rightarrow I / I^{2}$, définie par $d(a)=a \otimes 1-1 \otimes a$, est une dérivation et toute dérivation de $A$ à valeurs dans un $A$-module se factorise de façon unique à travers $d$. Il existe un prolongement unique de $d$ en une antidérivation $K$-linéaire, de carré nul, de l'algèbre extérieure $\wedge_{A}\left(I / I^{2}\right)$, qui fait de cette algèbre un complexe différentiel gradué, appelé complexe de de Rham algébrique. On peut considérer que c'est ce complexe qui définit la "structure différentielle" du schéma $\operatorname{Spec}(A)$. Si l'algèbre $A$ n'est pas commutative, cette construction ne se généralise pas. En effet, alors $I$ n'est plus un idéal de $A \otimes A$, 
mais seulement un $(A, A)$-bimodule, et en particulier, $I^{2} \not \subset I$ et on ne peut donc même pas considérer $I / I^{2}$. On peut néanmoins, définir une application $K$-linéaire $d: A \rightarrow I$, par $d(a)=a \otimes 1-1 \otimes a$, qui est une dérivation, universelle parmi les dérivations de $A$ à valeurs dans un bimodule, et qui se prolonge en une antidérivation $K$-linéaire, de carré nul, unique de l'algèbre tensorielle

$$
T_{A}(I)=\bigoplus_{n \in \mathbb{N}} \underbrace{I \otimes_{A} I \otimes_{A} \ldots \otimes_{A} I}_{n \text { fois }}
$$

du bimodule $I$, qui fait de $T_{A}(I)$ un complexe différentiel gradué, appelé complexe de de Rham universel [Co, Ka]. Ce complexe ne constitue pas une généralisation du complexe de de Rham algébrique. En effet, si l'algèbre $A$ est commutative, il est en général beaucoup plus gros que ce dernier. En plus, il ne paraît être un bon substitut du complexe de de Rham algébrique que pour des algèbres "très loin du cas commutatif" (comme les algèbres associatives libres, ou les algèbres de matrices).

Idéalement, pour généraliser la notion de complexe de de Rham algébrique, au cas non commutatif, on voudrait associer fonctoriellement à toute algèbre associative unifère, un complexe différentiel gradué, de sorte que l'on obtienne le complexe de de Rham universel, dans le cas d'une algèbre associative libre, et le complexe de de Rham algébrique, dans le cas d'une algèbre commutative. Cela paraît impossible, du moins de "façon canonique." La philosophie de cet article est que la donnée de "l'algèbre des fonctions" sur un "espace non commutatif," ou "quantique," ne suffit pas pour déterminer la structure différentielle de ce dernier, mais qu'il faut, pour cela, se donner en plus un complexe différentiel gradué, appelé complexe de de Rham, considéré comme faisant partie des données définissant la structure de cet espace. A une même algèbre plusieurs telles structures peuvent correspondre (on verra même des exemples de structures différentielles "non commutatives" sur une algèbre commutative). On définira donc un espace quantique, comme étant un objet de la catégorie opposée à celle des $K$-algèbres "munies d'un complexe de de Rham."

La motivation d'origine de mon travail vient du livre de Manin cité ci-dessus. Dans ce livre, Manin associe naturellement à toute algèbre quadratique graduée, considérée comme un espace linéaire quantique, un groupe linéaire quantique pouvant être interprété comme un "objet d'automorphismes linéaires" de cet espace. Pour obtenir par cette construction le groupe linéaire classique, dans le cas commutatif, ou le groupe quantique usuel $G L_{q}$ ( $q$-déformation du groupe linéaire "commutatif"), dans le cas quantique, il faut "ajouter les relations manquantes" ("add missing relations"), que Manin obtient moyennant une identification symétrique de l'espace vectoriel engendré par les générateurs de l'algèbre quadratique, à son dual. Le groupe quantique qu'il obtient dépend de cette identification. Ainsi, Manin parle de groupe "cryptoorthogonal" car il revient au même de se fixer une forme bilinéaire symétrique non dégénérée et d'imposer qu'elle reste invariante par les "automorphismes." Dans cet article, on interprète les relations manquantes de Manin autrement. Elles proviennent simplement du fait qu'on impose que les "automorphismes" respectent la structure différentielle qu'on se donne. La plupart des résultats de Manin s'étendent sans difficulté dans ce nouveau contexte, en modifiant à peine les démonstrations. (Après la fin de cette rédaction, j'ai reçu un preprint de Manin, où il adopte le point de vue exposé ici [Man4]).

Dans le premier paragraphe, on développe les préliminaires relatifs aux algèbres différentielles graduées. Au paragraphe deux, on introduit la notion d'espace quantique, ainsi que celle de cône quantique. On définit le produit de deux espaces quan- 
tiques, l'espace quantique opposé, les espaces quantiques de type fini, et les immersions fermées d'un espace quantique dans un autre. On étudie les propriétés élémentaires de ces espaces et on en donne quelques exemples. Dans le paragraphe trois, on introduit la notion de monoïde quantique, celle de monoïde quantique matriciel, on définit l'action d'un monoïde quantique sur un espace quantique et on démontre l'existence de l'espace quantique des morphismes d'un cône quantique dans un autre, généralisant un résultat de Manin. On obtient ainsi, comme cas particulier, le monoïde quantique des endomorphismes d'un cône quantique. On termine par quelques exemples de monoïdes quantiques, et on retrouve une construction analogue à celle du QISM, associant à une $R$-matrice, un monoïde quantique. Au paragraphe quatre, on introduit la notion de groupe quantique, et en généralisant une construction de Manin, on associe, de façon universelle, à tout monoïde quantique un groupe quantique. Cette construction est l'équivalent quantique du procédé qui associe à un monoïde, le groupe de ses éléments inversibles. Enfin, on termine par l'étude des monoïdes de Cramer, et l'exemple d'un calcul différentiel sur la déformation multiparamétrique du groupe linéaire, introduite par M. Artin, W. Shelter et J. Tate [AST].

Une version préliminaire de ce travail a été rédigée l'été 1989, sous forme d'une lettre adressée à J.-L. Verdier. J'ai appris sa mort tragique, avant que cette lettre ne soit postée. Un résumé a été publié aux C.R.A.S. [Mal1]. Le présent article est incontestablement influencé par le livre de Yu.I. Manin [Man2], par les articles de S.L. Woronowicz (qui est je pense à l'origine de l'idée de considérer un "calcul différentiel" comme étant une donnée supplémentaire dans la définition d'un espace non commutatif, ou "pseudoespace" [Wo1, Wo2], ainsi que par les exposés de P. Cartier au Séminaire de l'École Normale Supérieure, P. Cartier que je voudrais remercier pour les discussions utiles que j'ai pu avoir avec lui. Je remercie également Y. Kosmann-Schwarzbach, pour sa lecture attentive de mon manuscrit et ses nombreuses remarques. Je voudrais citer ici les noms des personnes qui ont travaillé sur le même sujet et qui ont obtenu indépendamment des résultats ou des exemples analogues, sans avoir eu une influence directe sur ce travail: D. Bernard, T. Brzeziński, U. Carow-Watamura, H. Dabrowski, E. Demidov, D. Gurevich, T. Hibi, B. Jurčo, E. Mukhin, F. Müller-Hoissen, M. Noumi, A. Radul, J. Rembieliński, M. Rosso, V. Rubtsov, A. Schirrmacher, M. Schlieker, W.B. Schmidke, B. Tsygan, T. Umeda, S.P. Vokos, M. Wakayama, S. Watamura, W. Weich, J. Wess, D. Zhdanovich et B. Zumino [Bd, Br, BDR, CSWW, GRR, HW, Ju, M-H, NUW, Ro, Sch, SWZ, SVZ, Tsy, WZ]. Dans une direction différente, on doit mentionner le travail de K. Aomoto [Ao1, Ao2, Ao3], de M. Dubois-Violette, R. Kerner et J. Madore [DV1, DV2, DKM1, DKM2], ainsi que le point de vue très original de S. Zakrzewski [Za2]. Enfin, on ne peut parler de calcul différentiel non commutatif sans citer A. Connes, bien que son point de vue soit totalement différent de celui adopté ici, et les adeptes de la cohomologie cyclique [Co, FT, Ka, Kas, MNW1, MNW2, Ta]. Je voudrais présenter mes excuses à tous ceux dont j'oublie de mentionner le travail. Ce n'est que par simple ignorance.

\section{Algèbres différentielles}

Dans cet article, $K$ désigne un anneau commutatif. Toutes les algèbres considérées seront des $K$-algèbres associatives unifères, non nécessairement commutatives, et les morphismes d'algèbres seront unifères. On notera $\otimes$ le produit tensoriel $\otimes_{K}$ sur $K$. Si $I$ désigne un ensemble et pour tout $i, i \in I, x_{\imath}$ l'image canonique de $i$ dans 
l'algèbre associative unifère libre construite sur $I$ ([Bo], A III, p. 21), on notera cette dernière $K\left\langle\left(x_{i}\right)_{i \in I}\right\rangle$, et on dira qu'elle est l'algèbre des polynômes non-commutatifs en les indéterminées $\left(x_{\imath}\right)_{i \in I}$.

\subsection{Bimodule des différentielles non commutatives}

(1.1.1) Soient $A$ une $K$-algèbre et $M$ un $(A, A)$-bimodule ([Bo], A III p. 38-39). On rappelle qu'une $K$-dérivation de $A$ dans $M$ est une application $K$-linéaire $D: A \rightarrow M$ telle que

$$
\forall a, a^{\prime} \in A \quad D\left(a \cdot a^{\prime}\right)=(D a) \cdot a^{\prime}+a \cdot\left(D a^{\prime}\right) .
$$

On dit que $M$ est engendré par l' image de $D$, si $D(A)$ engendre $M$ comme $A$-module à gauche (ou comme $A$-module à droite, ou comme $(A, A)$-bimodule, conditions qui sont équivalentes).

(1.1.2) Soit $\mu: A \otimes A \rightarrow A$ l'application $K$-linéaire définie par la multiplication de $A(\mu(a \otimes b)=a \cdot b)$. Alors $\mu$ est un morphisme de $(A, A)$-bimodules et en particulier le noyau $I=\operatorname{Ker}(\mu)$ est un $(A, A)$-bimodule (bimodule des "différentielles non commutatives" de $A$ ) et l'application $K$-linéaire $d: A \rightarrow I$ définie par

$$
d(a)=a \otimes 1-1 \otimes a
$$

est une $K$-dérivation dont l'image engendre $I$ et qui possède la propriété universelle suivante: pout tout $(A, A)$-bimodule $M$ et toute $K$-dérivation $D: A \rightarrow M$ il existe un morphisme unique de $(A, A)$-bimodules $h: I \rightarrow M$ tel que $D=h \circ d$ ([Bo], A III, p. 132).

(1.1.3) On rappelle que dans le cas où l'algèbre $A$ est commutative, $\mu$ est un morphisme de $K$-algèbres, $I$ est un idéal de $A \otimes A$ et si l'on pose $\Omega^{1}=I / I^{2}$ (module des différentielles ordinaires), les deux structures de $A$-module sur $\Omega^{1}$ déduites de la structure de bimodule de $I$ sont identiques et le composé de $d$ avec la surjection canonique $I \rightarrow \Omega^{1}$ (composé qu'on désignera aussi par $d$ ) est une $K$-dérivation jouissant de la propriété universelle suivante: pour tout $A$-module $M$ et toute $K$ dérivation $D: A \rightarrow M$ (pour la structure de $(A, A)$-bimodule de $M$ déduite de sa structure de $A$-module) il existe une application $A$-linéaire unique $h: \Omega^{1} \rightarrow M$ telle que $D=h \circ d$ ([Bo], A III, p. 133-134).

\subsection{Algèbres différentielles graduées}

Définition (1.2.1) On appelle algèbre différentielle graduée, ou plus simplement algèbre différentielle, une $K$-algèbre $\mathbb{N}$-graduée

$$
\Omega=\bigoplus_{n \in \mathbb{N}} \Omega^{n}
$$

munie d'une application $K$-linéaire $d$ (la différentielle) homogène de degré 1 ,

$$
d: \Omega \rightarrow \Omega, \quad d=\bigoplus_{n \in \mathbb{N}} d_{n}, \quad d_{n}: \Omega^{n} \rightarrow \Omega^{n+1}
$$

telle que:

i) $d^{2}=0$;

ii) $d(a \cdot b)=d a \cdot b+(-1)^{n} a \cdot d b, \quad a \in \Omega^{n}, b \in \Omega$. 
Un morphisme d'algèbres différentielles est un morphisme de $K$-algèbres graduées, de degré 0 , qui commute aux différentielles. On dit qu'une algèbre différentielle $(\Omega, d)$ est engendrée par ses éléments de degré 0 , si la $K$-algèbre sous-jacente à $\Omega$ est engendrée par $\Omega^{0} \cup d \Omega^{0}$. On dit qu'une partie $J$ de $\Omega$ est un idéal différentiel (gradué) si $J$ est un idéal bilatère gradué de $\Omega$ et $d(J) \subset J$. Pour toute partie de $\Omega$ il existe un plus petit idéal différentiel la contenant, appelé idéal différentiel engendré par cette partie. Si $S$ désigne une partie de $\Omega$ formée d'éléments homogènes, l'idéal différentiel engendré par $S$ est égal à l'idéal bilatère engendré par $S \cup d(S)$.

Exemple (1.2.2). Soient $I$ un ensemble et $\Omega=K\left\langle\left(x_{i}\right)_{i \in I},\left(\xi_{i}\right)_{i \in I}\right\rangle$ l'algèbre des polynômes non-commutatifs en les indéterminées $x_{i}$ et $\xi_{i}$. On considère la graduation de type $\mathbb{N}$ sur $\Omega$ définie par le degré total en les $\left(\xi_{i}\right)_{i \in I}$. On définit une différentielle $d$ sur $\Omega$ par $d x_{i}=\xi_{i}$, pour $i \in I$, et les propriétés (i) et (ii) de la définition (1.2.1). Alors $\Omega$ est une $K$-algèbre différentielle graduée appelée $K$-algèbre différentielle libre engendrée par la famille des indéterminées $\left(x_{i}\right)_{i \in I}$ et elle est notée $K\left\langle\left\langle\left(x_{i}\right)_{i \in I}\right\rangle\right\rangle$. Elle est engendrée par ses éléments de degré 0 et satisfait à la propriété universelle suivante: pour toute $K$-algèbre différentielle graduée $\Omega^{\prime}$ et toute famille $\left(a_{\imath}\right)_{\imath \in I}$ d'éléments de $\Omega^{\prime \prime}$ il existe un morphisme unique $f: \Omega \rightarrow \Omega^{\prime}$ de $K$-algèbres différentielles tel que $f\left(x_{i}\right)=a_{i}$, pour $i \in I$.

Théorème (1.2.3). Soient $A$ une $K$-algèbre, $M$ un $(A, A)$-bimodule et $D: A \rightarrow M$ une $K$-dérivation telle que $M$ soit engendré par l'image de $D$. Alors il existe une $K$-algèbre différentielle $\left(\Omega_{D}, d\right)$, engendrée par ses éléments de degré 0 , telle que $\Omega_{D}^{0}=A, \Omega_{D}^{1}=M, d_{0}=D$ et satisfaisant à la propriété universelle suivante: pour toute $K$-algèbre différentielle $\left(\Omega^{\prime}, d^{\prime}\right)$ et tout morphisme de $K$-algèbres $\varrho: A \rightarrow \Omega^{\prime 0}$ tel qu'il existe un morphisme (nécessairement unique) de $(A, A)$-bimodules $\sigma: M \rightarrow \Omega^{\prime 1}$ tel que $d_{0}^{\prime} \circ \varrho=\sigma \circ D$, il existe un morphisme unique de $K$-algèbres différentielles $f: \Omega_{D} \rightarrow \Omega^{\prime}$ tel que $f_{0}=\varrho\left(\right.$ et alors $\left.f_{1}=\sigma\right)$.

Démonstration. Soient $\left(a_{i}\right)_{i \in I}$ un système de générateurs de la $K$-algèbre $A$ et $\Omega=K\left\langle\left\langle\left(x_{i}\right)_{i \in I}\right\rangle\right\rangle$ l'algèbre différentielle libre construite sur $I$. La $K$-algèbre $\Omega^{0}$ s'identifie canoniquement à l'algèbre des polynômes non-commutatifs $K\left\langle\left(x_{\imath}\right)_{i \in I}\right\rangle$ et le $\left(\Omega^{0}, \Omega^{0}\right)$-bimodule $\Omega^{1}$ est canoniquement isomorphe au bimodule $\Omega^{0} \otimes K^{(I)} \otimes \Omega^{0}$, où $K^{(I)}$ désigne le $K$-module libre de base $\left(d x_{i}\right)_{i \in I}$. Il existe donc un morphisme unique de $K$-algèbres $p_{0}: \Omega^{0} \rightarrow A$ (resp. de $\left(\Omega^{0}, \Omega^{0}\right)$-bimodules $p_{1}: \Omega^{1} \rightarrow M$ ) tel que $p_{0}\left(x_{i}\right)=a_{i}$ (resp. $\left.p_{1}\left(d x_{i}\right)=D a_{i}\right)$, pour $i \in I$. On a $p_{1} \circ d=D \circ p_{0}$ et si l'on pose $J_{0}=\operatorname{Ker}\left(p_{0}\right)$ et $J_{1}=\operatorname{Ker}\left(p_{1}\right), d\left(J_{0}\right) \subset J_{1}$. L'idéal différentiel $J$ de $\Omega$ engendré par $J_{0} \cup J_{1}$ est donc l'idéal bilatère de $\Omega$ engendré par $J_{0} \cup J_{1} \cup d\left(J_{1}\right)$. On en déduit que si l'on pose $\Omega_{D}=\Omega / J$, le morphisme $p_{0}$ (resp. $p_{1}$ ) induit un isomorphisme de $\Omega_{D}^{0}$ (resp. $\Omega_{D}^{1}$ ) sur $A$ (resp. $M$ ) (car la famille $\left(a_{i}\right)_{i \in I}$ engendre $A$ et l'image de $D$ engendre $M$ ) et ces isomorphismes identifient $d_{0}$ à $D$. La propriété universelle de $\Omega_{D}$ résulte aussitôt de celle de $\Omega$.

Corollaire (1.2.4). Soit A une $K$-algèbre. Il existe une $K$-algèbre différentielle $(\Omega, d)$ telle que $\Omega^{0}=A$, satisfaisant à la propriété universelle suivante: pour toute $K$ algèbre différentielle $\left(\Omega^{\prime}, d^{\prime}\right)$ et tout morphisme de $K$-algèbres $\varrho: A \rightarrow \Omega^{\prime 0}$, il existe un morphisme unique de K-algèbres différentielles $f: \Omega \rightarrow \Omega^{\prime}$ tel que $f_{0}=\varrho$.

Démonstration. Le corolaire résulte du théorème (1.2.3) appliqué au bimodule $I$ des différentielles non commutatives et à la dérivation universelle $d: A \rightarrow I$ définis dans (1.1.2). 
Cette algèbre différentielle s'appelle complexe de de Rham universel. Plus généralement, on appelle complexe de de Rham sur une $K$-algèbre $A$, une $K$-algèbre différentielle $\Omega$ telle que:

a) $\Omega^{0}=A$;

b) $\Omega$ est engendrée par ses éléments de degré 0 [cf. déf. (1.2.1)].

Il résulte du corollaire (1.2.4) que tout complexe de de Rham est quotient du complexe de de Rham universel, et le théorème (1.2.3) permet d'associer à tout ( $A, A$ )-bimodule $M$ et toute dérivation $D: A \rightarrow M$, dont l'image engendre $M$, un complexe de de Rham sur $A$.

Exemple (1.2.5). Le complexe de de Rham universel de l'algèbre des polynômes non commutatifs $K\left\langle\left(x_{i}\right)_{i \in I}\right\rangle$ est isomorphe à algèbre différentielle libre $K\left\langle\left\langle\left(x_{i}\right)_{i \in I}\right\rangle\right\rangle$ engendrée par la famille des indéterminées $\left(x_{i}\right)_{i \in I}$.

Exemple (1.2.6). Considérons une partie $S$ de l'algèbre des polynômes non-commutatifs $K\left\langle\left(x_{i}\right)_{i \in I}\right\rangle$ et soit $A$ l'algèbre quotient de $K\left\langle\left(x_{i}\right)_{i \in I}\right\rangle$ par l'idéal bilatère engendré par $S$. Alors le complexe de de Rham universel de $A$ est isomorphe au quotient de l'algèbre différentielle libre $K\left\langle\left\langle\left(x_{i}\right)_{i \in I}\right\rangle\right\rangle$ par l'idéal différentiel engendré par $S$, autrement dit, l'idéal bilatère engendré par $S \cup d(S)$.

Exemple (1.2.7). Soient $A$ une $K$-algèbre commutative, $M=I / I^{2}$ le module des différentielles ordinaires et $D: A \rightarrow M$ la dérivation universelle (cf. 1.1.3). Alors si 2 est inversible dans $K$, le complexe de de Rham associé à $D$ par le théorème (1.2.3) est le complexe de de Rham algébrique habituel (cf. [EGA IV $\left.{ }_{4}\right]$ 16.6.2, p. 34) et il est en général distinct du complexe de de Rham universel de $A$.

\section{Espaces quantiques}

\subsection{La catégorie des espaces quantiques}

Définition (2.1.1). La catégorie des K-espaces quantiques est la catégorie opposée à la catégorie des $K$-algèbres différentielles graduées, engendrées par les éléments de degré 0 . On appelle $K$-espace quantique, ou plus simplement espace quantique (resp. morphisme d'espaces quantiques) un objet (resp. un morphisme) de cette catégorie.

La catégorie des espaces quantiques est donc munie d'un foncteur contravariant canonique, associant à un espace quantique $X$ une algèbre différentielle notée $\Omega_{X}$ et appelée complexe de de Rham de $X$ et à tout morphisme d'espaces quantiques $f: X \rightarrow Y$ un morphisme d'algèbres différentielles graduées $f^{*}: \Omega_{Y} \rightarrow \Omega_{X}$. Ce foncteur est un isomorphisme de la catégorie opposeé à celle des espaces quantiques sur la catégorie des algèbres différentielles graduées engendrées par les éléments de degré 0. L'isomorphisme inverse sera noté Spec. Le foncteur Spec associe donc à toute algèbre différentielle graduée $\Omega$ engendrée par ses éléments de degré 0 , un espace quantique $\operatorname{Spec}(\Omega)$ et à tout morphisme d'algèbres différentielles $\varphi: \Omega \rightarrow \Omega^{\prime}$ un morphisme d'espaces quantiques $\operatorname{Spec}(\varphi): \operatorname{Spec}\left(\Omega^{\prime}\right) \rightarrow \operatorname{Spec}(\Omega)$. En particulier, on note $\operatorname{Spec}(K)$ l'espace quantique associé à l'algèbre différentielle $K$, munie de la différentielle identiquement nulle. Pour tout espace quantique $X$ on note $\mathscr{O}_{X}$ l'algèbre $\Omega_{X}^{0}$ des éléments de degré 0 de $\Omega_{X}$, qu'on appelle algèbre des fonctions sur $X$ (ou algèbre des observables sur l'espace quantique $X$ ), et pour tout morphisme d'espaces quantiques $f: X \rightarrow Y$ on note aussi $f^{*}: \mathscr{Q}_{Y} \rightarrow \mathscr{Q}_{X}$ la composante de degré $0 \mathrm{du}$ morphisme $f^{*}: \Omega_{Y} \rightarrow \Omega_{X}$. 
On dit qu'un espace quantique $X$ est simple, s'il existe une $K$-algèbre $A$, un $(A, A)$-bimodule $M$ et une $K$-dérivation $D: A \rightarrow M$ dont l'image engendre $M$ tels que l'espace quantique $X$ soit isomorphe à $\operatorname{Spec}\left(\Omega_{D}\right)$, où $\Omega_{D}$ désigne l'algèbre différentielle définie dans le théorème (1.2.3).

Proposition (2.1.2). Soient $X$ un espace quantique, $\left(a_{i}\right)_{i \in I}$ une famille d'éléments de $\mathscr{\odot}_{X}$ qui engendrent l'algèbre différentielle $\Omega_{X}, \Omega=K\left\langle\left\langle\left(x_{i}\right)_{i \in I}\right\rangle\right\rangle$ la $K$-algèbre différentielle libre engendrée par la famille des indéterminées $\left(x_{i}\right)_{i \in I}, \varphi: \Omega \rightarrow \Omega_{X}$ le morphisme (surjectif) de K-algèbres différentielles tel que $\varphi\left(x_{i}\right)=a_{i}$, pour $i \in I$, [cf. (1.2.2.)] et $J=\bigoplus_{n \in \mathbb{N}} J^{n}$ le noyau de $\varphi$. Pour que l'espace quantique $X$ soit simple il faut et il suffit que $J$ soit l'idéal différentiel de $\Omega$ engendré par $J^{0} \cup J^{1}$.

La démonstration de cette proposition est laissée au lecteur. Elle s'inspire directement de la démonstration du théorème (1.2.3).

\subsection{Sous-espaces quantiques et espace quantique opposé}

Définition (2.2.1). Soit $X$ un espace quantique. On dit qu'un espace quantique $X^{\prime}$ est un sous-espace quantique fermé de $X$ s'il existe un idéal différentiel $\mathscr{T}$ de $\Omega_{X}$ engendré par ses éléments homogènes de degré 0 (comme idéal différentiel) tel que $\Omega_{X^{\prime}}=\Omega_{X} / \mathscr{T}$, et on dit que le morphisme $i: X^{\prime} \rightarrow X$ déduit de la surjection canonique $\Omega_{X} \rightarrow \Omega_{X^{\prime}}$ est l'immersion (fermée) canonique de $X^{\prime}$ dans $X$. On dit qu'un morphisme d'espaces quantiques $f: Y \rightarrow X$ est une immersion fermée, s'il existe un sous-espace quantique fermé $X^{\prime}$ de $X$ et un isomorphisme $g: Y \rightarrow X^{\prime}$, tels que $f=i \circ g$, où $i: X^{\prime} \hookrightarrow X$ désigne l'immersion canonique.

(2.2.2) Soit $\Omega$ une $K$-algèbre différentielle. On appelle algèbre différentielle opposée et on note $\Omega^{\text {opp }}$ l'algèbre différentielle dont le $K$-module gradué sous-jacent et la différentielle sont ceux de $\Omega$ et dont la multiplication o est définie par

$$
a \circ b=(-1)^{m n} b a, \quad a \in \Omega^{m}, b \in \Omega^{n} .
$$

On définit ainsi un foncteur de la catégorie des $K$-algèbres différentielles dans ellemême et on remarque que si $\Omega$ est engendrée par ses éléments de degré 0 il en est de même pour $\Omega^{\mathrm{opp}}$. On en déduit un foncteur de la catégorie des espaces quantiques dans elle-même qui est une involution.

Si $X$ (resp. $f: X^{\prime} \rightarrow X$ ) désigne un espace quantique (resp. un morphisme d'espaces quantiques) on note $X^{\text {opp }}$ (resp. $f^{\text {opp}) ~ s o n ~ i m a g e ~ p a r ~ c e t t e ~ i n v o l u t i o n ~ e t ~}$ on a donc $X^{\text {opp }}=\operatorname{Spec}\left(\Omega_{X}^{\mathrm{opp}}\right)$. On dit que $X^{\text {opp }}$ est l'espace quantique opposé à $X$.

\subsection{Produit d'espaces quantiques}

(2.3.1) Soit $S$ un espace quantique. Pour tout espace quantique $X$ on appelle $S$-point de $X$ un morphisme d'espaces quantiques de $S$ dans $X$.

Définition (2.3.2). Soient $S, X, X^{\prime}$ des espaces quantiques et $f: S \rightarrow X$ et $f^{\prime}: S \rightarrow X^{\prime}$ deux $S$-points de $X$ et $X^{\prime}$ respectivement. On dit que $f$ et $f^{\prime}$ sont simultanément observables si $\operatorname{Im}\left(f^{*}\right)$ et $\operatorname{Im}\left(f^{\prime *}\right)$ commutent au sens gradué (élément par élément), autrement dit, si pour tout $a \in \operatorname{Im}\left(f^{*}\right) \cap \Omega_{S}^{m}$ et tout $b \in \operatorname{Im}\left(f^{\prime *}\right) \cap \Omega_{S}^{n}$ on a

$$
a b=(-1)^{m n} b a .
$$


Si la relation "être simultanément observable" est bien symétrique, elle n'est ni réflexive ni transitive et en particulier, elle n' est pas une relation d'équivalence comme cette terminologie pourait le faire croire. Cette terminologie vient de la physique. En effet, on dit que deux éléments $a$ et $b$ de l'algèbre des observables $\mathscr{C}_{S}$ d'un espace quantique $S$ [cf. (2.1.1)] sont simultanément observables si $a$ et $b$ commutent.

(2.3.3) On va définir un produit d'espaces quantiques qui ne sera pas le produit au sens des catégories (qui existe mais qui ne nous intéressera pas dans cet article). Le produit catégorique de deux espaces quantiques $X$ et $Y$ représente le foncteur contravariant qui à un espace quantique $S$ associe l'ensemble des couples de $S$-points de $X$ et $Y$. Le produit que nous allons définir représentera le foncteur associant l'ensemble des couples formés de $S$-points simultanément observables. Du point de vue de la physique, on peut dire que le produit de deux espaces quantiques représentera le système quantique formé par la juxtaposition des deux systèmes, sans interactions entre eux.

(2.3.4) Soient $\Omega^{\prime}$ et $\Omega^{\prime \prime}$ deux $K$-algèbres différentielles graduées. Le produit tensoriel $\Omega$ de $\Omega^{\prime}$ et $\Omega^{\prime \prime}$ est l'algèbre différentielle graduée définie comme suit:

a) comme $K$-module $\Omega=\Omega^{\prime} \otimes \Omega^{\prime \prime}$;

b) comme $K$-algèbre $\Omega=\Omega^{\prime} g_{\otimes} \Omega^{\prime \prime}$ ([Bo], A III, p. 49), autrement dit, $\left(a \otimes a^{\prime}\right) \cdot\left(b \otimes b^{\prime}\right)=(-1)^{m n} a b \otimes a^{\prime} b^{\prime}, \quad a \in \Omega^{\prime}, \quad a^{\prime} \in \Omega^{\prime \prime m}, \quad b \in \Omega^{\prime n}, \quad b^{\prime} \in \Omega^{\prime \prime}$

la graduation étant définie par

$$
\Omega^{m}=\bigoplus_{\substack{m^{\prime}, m^{\prime \prime} \geq 0 \\ m^{\prime}+m^{\prime \prime}=m}} \Omega^{\prime m^{\prime}} \otimes \Omega^{\prime \prime m^{\prime \prime}} ;
$$

c) la différentielle $d$ de $\Omega$ est définie par

$$
d\left(a \otimes a^{\prime}\right)=d a \otimes a^{\prime}+(-1)^{m} a \otimes d a^{\prime}, \quad a \in \Omega^{\prime m}, \quad a^{\prime} \in \Omega^{\prime \prime} .
$$

Le produit tensoriel des algèbres différentielles graduées $\Omega^{\prime}$ et $\Omega^{\prime \prime}$ est noté $\Omega^{\prime}{ }^{g} \otimes \Omega^{\prime \prime}$. Si $\Omega^{\prime}$ et $\Omega^{\prime \prime}$ sont engendrées par les éléments de degré 0 il en est de même pour $\Omega$.

Définition (2.3.5). Soient $X$ et $Y$ deux espaces quantiques. On appelle produit de $X$ et $Y$, et on note $X \times Y$, l'espace quantique $\operatorname{Spec}\left(\Omega_{X}{ }^{g} \otimes \Omega_{Y}\right)$.

L'application canonique $\Omega_{X} \rightarrow \Omega_{X}{ }^{g} \otimes \Omega_{Y}$ (resp. $\Omega_{Y} \rightarrow \Omega_{X}{ }^{g} \otimes \Omega_{Y}$ ) définie par $a \mapsto a \otimes 1$ (resp. $a^{\prime} \mapsto 1 \otimes a^{\prime}$ ) définit un morphisme d'espaces quantiques $X \times Y \rightarrow X$ (resp. $X \times Y \rightarrow Y$ ) appelé première (resp. deuxième) projection.

Proposition (2.3.6). Soient $X$ et $Y$ deux espaces quantiques, $\mathrm{pr}_{1}: X \times Y \rightarrow X$ (resp. $\mathrm{pr}_{2}: X \times Y \rightarrow Y$ ) la première (resp. la deuxième) projection. Pour tout espace quantique $S$ l'application qui associe à un $S$-point $f: S \rightarrow X \times Y$ le couple $\left(\mathrm{pr}_{1} \circ f, \mathrm{pr}_{2} \circ f\right)$ établi une bijection entre les $S$-points de $X \times Y$ et les couples formés de $S$-points simultanément observables de $X$ et $Y$.

(2.3.7) Le produit d'espaces quantiques définit un bifoncteur et si $X, Y$ et $Z$ désignent des espaces quantiques il existe des isomorphismes fonctoriels canoniques

$$
\left.\begin{array}{rl}
(X \times Y) \times Z \rightarrow X \times(Y \times Z) & \text { (contrainte d'associativité) } \\
X \times Y \rightarrow Y \times X & \text { (contrainte de commutativité) } \\
X \times \operatorname{Spec}(K) \rightarrow X \\
\operatorname{Spec}(K) \times X \rightarrow X
\end{array}\right\} \quad \text { (contraintes d'unité) }
$$


satisfaisant aux conditions de compatibilité des catégories tensorielles symétriques ([DM], p. 104-105). La seule précision utile concerne la contrainte de commutativité: l'isomorphisme $s: X \times Y \rightarrow Y \times X$ est égal à $\operatorname{Spec}(\sigma)$, où $\sigma: \Omega_{Y}{ }^{g} \otimes \Omega_{X} \rightarrow$ $\Omega_{X}{ }^{g} \otimes \Omega_{Y}$ est défini par

$$
\sigma\left(a \otimes a^{\prime}\right)=(-1)^{m, m^{\prime}} a^{\prime} \otimes a, \quad a \in \Omega_{Y}^{m}, \quad a^{\prime} \in \Omega_{X}^{m^{\prime}} .
$$

\subsection{Cônes quantiques}

Définition (2.4.1). On appelle cône quantique un espace quantique $X$, dont le complexe de de Rham $\Omega_{X}$ est muni d'une bigradatuation compatible avec la graduation d'algèbre différentielle de $\Omega_{X}$, autrement dit, pour tout $n, n \in \mathbb{N}$, d'une décomposition en somme directe de $K$-modules

$$
\Omega_{X}^{n}=\bigoplus_{m \in \mathbb{N}} \Omega_{X}^{m, n}
$$

satisfaisant aux propriétés suivantes:

i) $\Omega_{X}^{0,0}=K$

ii) $\Omega_{X}^{0}$ est engendré par $\Omega_{X}^{1,0}$ comme $K$-algèbre;

iii) la multiplication est compatible avec la bigraduation:

$$
\Omega_{X}^{m, n} \cdot \Omega_{X}^{m^{\prime}, n^{\prime}} \subset \Omega_{X}^{m+m^{\prime}, n+n^{\prime}} \quad m, n, m^{\prime}, n^{\prime} \in \mathbb{N} ;
$$

iv) la différentielle est bihomogène de bidegré $(-1,1)$ :

$$
d\left(\Omega_{X}^{m, n}\right) \subset \Omega_{X}^{m-1, n+1}, \quad m, n \in \mathbb{N} .
$$

Un morphisme de cônes quantiques est un morphisme d'espaces quantiques $f: X \rightarrow Y$ tel que $f^{*}$ soit bihomogène de bidegré $(0,0)$ :

$$
f^{*}\left(\Omega_{Y}^{m, n}\right) \subset \Omega_{X}^{m, n}, \quad m, n \in \mathbb{N} .
$$

Il est facile de vérifier que pour cela il suffit que

$$
f^{*}\left(\Omega_{Y}^{1,0}\right) \subset \Omega_{X}^{1,0} .
$$

Exemple (2.4.2). Soit $\Omega=K\left\langle\left\langle\left(x_{i}\right)_{i \in I}\right\rangle\right\rangle$ l'algèbre différentielle libre engendrée par la famille des indéterminées $\left(x_{i}\right)_{i \in I}$ [cf. (1.2.2)]. On définit une bigraduation sur $\Omega$ par le "degré total en les $\left(x_{i}\right)_{i \in I}$ " et le "degré total en les $\left(\xi_{i}\right)_{i \in I}$," où $\xi_{i}=d x_{i}$, pour $i \in I$. L'espace quantique $\operatorname{Spec}(\Omega)$ est ainsi muni d'une structure de cône quantique. On dit que $X$ est le cône quantique associé à l'ensemble $I$, qu'on note $C(I)$. Si $J$ désigne un idéal différentiel bigradué de $\Omega$, l'algèbre différentielle quotient $\Omega / J$ est munie d'une bigraduation et $\operatorname{Spec}(\Omega / J)$ d'une structure de cône quantique. Tout cône quantique est isomorphe à un cône ainsi défini.

\subsection{Espaces quantiques de types fini}

Définition (2.5.1). On dit qu'un $K$-espace quantique $X$ est de type fini si la $K$-algèbre $\mathscr{Q}_{X}$ est de type fini. On dit qu'un cône quantique est de type fini si l'espace quantique sous-jacent 1'est. 
Exercise (2.5.2). Soit $X$ un espace quantique. Les conditions suivantes sont équivalentes:

i) $X$ est de type fini;

ii) $\Omega_{X}$ est une $K$-algèbre de type fini;

iii) $\Omega_{X}$ est engendrée comme $K$-algèbre différentielle par un nombre fini d'éléments.

De plus, si $X$ est un cône quantique ces conditions sont équivalentes à:

iv) $\Omega_{X}^{1,0}$ est un $K$-module de type fini.

\subsection{Exemples d'espaces et de cônes quantiques}

Dans les exemples suivants, si $\Omega$ désigne une algèbre différentielle graduée et $a_{1}, \ldots, a_{n}$ des éléments hommogènes de $\Omega$, on désigne par $\left(a_{1}, \ldots, a_{n}\right)$ (resp. $\left.\left(\left(a_{1}, \ldots, a_{n}\right)\right)\right)$ l'idéal bilatère (resp. l'idéal différentiel) engendré par ces éléments.

Exemple (2.6.1) (P. Cartier [Ca]).

$$
X=\operatorname{Spec}(K\langle\langle x\rangle\rangle /((\xi x-q x \xi))),
$$

où $\xi=d x$ et $q$ désigne un élément de l'anneau $K$. L'algèbre des observables de $X$ est isomorphe à l'algèbre des polynômes commutatifs $K[x]$. On peut donc considérer que l'espace $X$ est la droite affine ("commutative") munie d'une structure différentielle non commutative. Si $K$ est un corps et $q \neq-1$ on a

$$
\Omega_{X}=K\langle\langle x\rangle\rangle /\left(\xi x-q x \xi, \xi^{2}\right)
$$

[cf. (1.2.1)]. Si $q=-1$

$$
\Omega_{X}=K\langle\langle x\rangle\rangle /(\xi x+x \xi)
$$

et l'espace quantique

$$
X^{\prime}=\operatorname{Spec}\left(K\langle\langle x\rangle\rangle /\left(\xi x+x \xi, \xi^{2}\right)\right)
$$

n'est pas un espace quantique simple [cf. (2.1.2)]. L'élément $\xi x-q x \xi$ de $K\langle\langle x\rangle\rangle$ étant bihomogène en $x$ et $\xi$, l'espace quantique $X$ est muni d'une structure de cône quantique [cf. (2.4.2)].

Exemple (2.6.2). (Plan quantique) $\mathscr{C}_{q, p}^{2}=\operatorname{Spec}(K\langle\langle x, y\rangle\rangle / J)$, où si l'on pose $\xi=d x$ et $\eta=d y, J$ désigne l'idéal bilatère engendré par

$$
\begin{gathered}
y x-q x y, \quad \xi x-p q x \xi, \quad \xi y-p y \xi, \quad \eta x-q x \eta-(p q-1) y \xi, \\
\eta y-p q y \eta, \quad \xi^{2}, \quad \eta^{2}, \quad \xi \eta+p \eta \xi \quad(p, q \in K) .
\end{gathered}
$$

L'idéal $J$ est bigradué (pour la bigraduation définie par le degré total en $x$ et $y$ et le degré total en $\xi$ et $\eta$ ) et l'espace $\mathscr{C}_{q, p}^{2}$ est ainsi muni d'une structure de cône quantique [cf. (2.4.2)]. L'espace quantique $\mathscr{C}_{q, p}^{2}$ est simple si et seulement si $p q+1$ est inversible dans $K$. En effet, si $p q+1$, est inversible on a

$$
J=((y x-q x y, \xi x-p q x \xi, \xi y-p y \xi, \eta y-p q y \eta)) .
$$

Un cas particulier intéressant est le cas où $p=q$ introduit par $\mathrm{P}$. Cartier [Ca]. Si $q=1$ et $p \neq 1$ l'espace quantique $\mathscr{A}_{q, p}^{2}$ est le plan affine (commutatif) muni d'une structure différentielle non commutative.

Exercise (2.6.3). Démontrer que si $K$ est un corps et $q, p, q^{\prime}, p^{\prime} \in K$ les espaces quantiques $\mathscr{b}_{q, p}^{2}$ et $\mathscr{A}_{q^{\prime}, p^{\prime}}^{2}$ sont isomorphes, si et seulement si

$$
\left(q^{\prime}=q \text { et } p^{\prime}=p\right) \quad \text { ou } \quad\left(p q=1 \text { et } p^{\prime}=q \text { et } q^{\prime}=p\right) .
$$


Exemple (2.6.4). (Plan quantique de Jordan)

$$
A_{\mathrm{Jord}, \lambda, \mu}^{2}=\operatorname{Spec}(K\langle\langle x, y\rangle\rangle / J),
$$

où, si l'on pose $\xi=d x$ et $\eta=d y, J$ désigne l'idéal bilatère engendré par

$$
\begin{gathered}
y x-x y-\lambda x^{2}, \quad \xi x-x \xi, \quad \xi y+\lambda x \xi-y \xi, \\
\eta x-\lambda x \xi-x \eta, \quad \eta y+\lambda \mu x \xi+\mu x \eta-\mu y \xi-y \eta \\
\xi^{2}, \quad \eta \xi+\xi \eta, \quad \eta^{2}-\mu \xi \eta \quad(\lambda, \mu \in K) .
\end{gathered}
$$

On peut y définir une structure de cône quantique comme dans l'exemple (2.6.2). L'espace quantique $\mathscr{C}_{\mathrm{Jord}, \lambda, \mu}^{2}$ est simple si et seulement si 2 est inversible dans $K$ et alors

$$
J=\left(\left(y x-x y-\lambda x^{2}, \xi x-x \xi, \xi y+\lambda x \xi-y \xi, \eta y+\lambda \mu x \xi+\mu x \eta-\mu y \xi-y \eta\right)\right) .
$$

Exercise (2.6.5). Démontrer que si $K$ est un corps et $\lambda, \mu, \lambda^{\prime}, \mu^{\prime} \in K$, les espaces quantiques $\mathscr{A}_{\mathrm{J} \text { ord }, \lambda, \mu}^{2}$ et $\mathscr{C}_{\mathrm{J} \text { ord }, \lambda^{\prime}, \mu^{\prime}}^{2}$ sont isomorphes si et seulement s'il existe $\varrho \in K^{*}$ tel que

$$
\lambda^{\prime}=\varrho \lambda \quad \text { et } \quad \mu^{\prime}=\varrho \mu .
$$

\section{Monoïdes quantiques}

\subsection{Monoïdes quantiques}

Définition (3.1.1). On appelle monoïde quantique un triplet $(X, m, e)$, où $X$ désigne un espace quantique et

$$
m: X \times X \rightarrow X \quad \text { et } \quad e: \operatorname{Spec}(K) \rightarrow X
$$

des morphismes d'espaces quantiques satisfaisant aux propriétés suivantes:

i) associativité: le diagramme

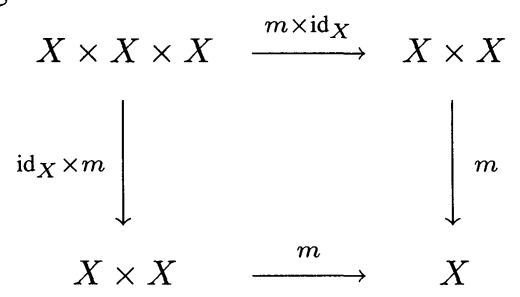

est commutatif (où l'on identifie $(X \times X) \times X$ à $X \times(X \times X)$ par la contrainte d'associativité);

ii) élément neutre: les diagrammes suivants sont commutatifs:
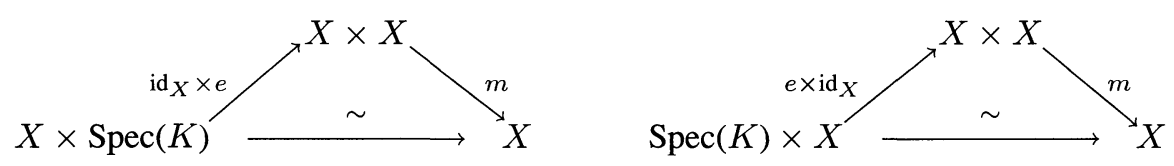

(où les isomorphismes $X \times \operatorname{Spec}(K) \stackrel{\sim}{\rightarrow} X$ et $\operatorname{Spec}(K) \times X \stackrel{\sim}{\rightarrow} X$ sont les contraintes d'unité). 
Si $(X, m, e)$ et $\left(X^{\prime}, m^{\prime}, e^{\prime}\right)$ sont des monoïdes quantiques, un morphisme de monoïdes quantiques de $(X, m, e)$ dans $\left(X^{\prime}, m^{\prime}, e^{\prime}\right)$ est un morphisme d'espaces quantiques

$$
f: X \rightarrow X^{\prime}
$$

rendant commutatifs les diagrammes suivants:

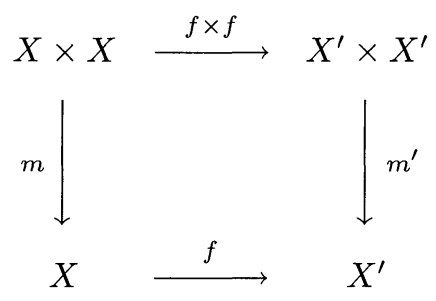

et

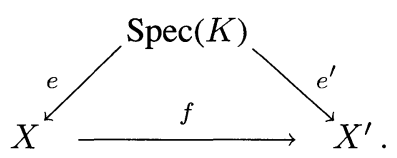

La notion de monoïde quantique est un cas particulier de la notion de monoïde dans une catégorie tensorielle.

Remarque (3.1.2). Soit $(X, m, e)$ un monoïde quantique. Alors $m^{*}$ et $e^{*}$ :

$$
m^{*}: \Omega_{X} \rightarrow \Omega_{X} g^{g} \Omega_{X}, \quad e^{*}: \Omega_{X} \rightarrow K
$$

sont respectivement le coproduit et la coünité d'une structure de $K$-bigèbre graduée gauche sur l'algèbre graduée sous-jacente à l'algèbre différentielle $\Omega_{X}$ ([Bo], A III, p. 148-149) et en particulier, la restriction de $m^{*}$ et $e^{*}$ à l'algèbre des observables $\mathscr{Q}_{X}:$

$$
m^{*}: \mathscr{Q}_{X} \rightarrow \mathscr{Q}_{X} \otimes \mathscr{Q}_{X}, \quad e^{*}: \mathscr{Q}_{X} \rightarrow K
$$

définit une structure de bigèbre sur cette algèbre. La condition de compatibilité de la différentielle $d_{X}$ de $\Omega_{X}$ avec le coproduit $m^{*}$, exprimant que $m$ est un morphisme d'espaces quantiques, se traduit comme suit: si

$$
m^{*}(a)=\sum_{i=1}^{n} a_{i}^{\prime} \otimes a_{\imath}^{\prime \prime}, \quad a \in \Omega_{X}, \quad a_{i}^{\prime} \in \Omega_{X}^{n_{i}}, \quad a_{i}^{\prime \prime} \in \Omega_{X},
$$

alors

$$
m^{*}\left(d_{X}(a)\right)=\sum_{i=1}^{n} d_{X} a_{i}^{\prime} \otimes a_{i}^{\prime \prime}+(-1)^{n_{\imath}} \sum_{i=1}^{n} a_{i}^{\prime} \otimes d_{X} a_{i}^{\prime \prime}
$$

On définit ainsi la notion de bigèbre différentielle graduée.

Exemple (3.1.3). Soient $\Omega=K\left\langle\left\langle\left(a_{j}^{i}\right)_{1 \leq i, j \leq n}\right\rangle\right\rangle$ l'algèbre différentielle libre engendrée par les indéterminées $\left(a_{j}^{i}\right)_{1<i, j \leq n}$ et $\Delta: \Omega \rightarrow \Omega^{g} \otimes \Omega$ et $\varepsilon: \Omega \rightarrow K$ les morphismes d'algèbres différentielles définis par 


$$
\Delta\left(a_{j}^{i}\right)=\sum_{k=1}^{n} a_{j}^{k} \otimes a_{k}^{i} \quad \text { et } \quad \varepsilon\left(a_{j}^{i}\right)=\delta_{j}^{i}, \quad 1 \leq i, j \leq n
$$

(où $\delta_{j}^{i}$ désigne le symbole de Kronecker). Alors $(\Omega, \Delta, \varepsilon)$ est une bigèbre différentielle graduée et $(\operatorname{Spec}(\Omega), \operatorname{Spec}(\Delta), \operatorname{Spec}(\varepsilon))$ un monoïde quantique.

\subsection{Monoïdes quantiques matriciels}

Définition (3.2.1). Soient $(X, m, e)$ un monoïde quantique et $\left(b_{j}^{i}\right)_{1 \leq i, j \leq n}$ une matrice à coefficients dans $\mathscr{O}_{X}$. On dit que $\left(b_{j}^{i}\right)_{1 \leq i, j \leq n}$ est une matrice multiplicative, si

$$
m^{*}\left(b_{j}^{i}\right)=\sum_{k=0}^{n} b_{j}^{k} \otimes b_{k}^{i} \quad \text { et } \quad e^{*}\left(b_{j}^{i}\right)=\delta_{j}^{i}, \quad 1 \leq i, j \leq n .
$$

On dit que $(X, m, e)$ est un monoïde quantique matriciel s'il possède une matrice multiplicative dont les coefficients engendrent la $K$-algèbre différentielle $\Omega_{X}$.

Proposition (3.2.2). Soit $(X, m, e)$ un monoïde quantique. Les conditions suivantes sont équivalentes:

i) $(X, m, e)$ est un monoïde quantique matriciel;

ii) il existe une famille finie de matrices multiplicatives dont les coefficients engendrent l'algèbre différentielle $\Omega_{X}$;

iii) $\left(\Omega_{X}, m^{*}, e^{*}\right)$ est une bigèbre différentielle quotient d' une bigèbre du type de celles définies dans l' exemple (3.1.3).

Démonstration. Les implications (i) $\Rightarrow$ (ii) et (iii) $\Rightarrow$ (i) sont évidentes. L'implication (i) $\Rightarrow$ (iii) résulte de la propriété universelle des algèbres différentielles libres. Pour démontrer que (ii) implique (i) il suffit de remarquer que si $\left(A_{i}\right)_{1 \leq i \leq n}$ est une famille finie de matrices multiplicatives alors la matrice

$$
A=\left(\begin{array}{cccc}
A_{1} & 0 & \ldots & 0 \\
0 & A_{2} & \ldots & 0 \\
\vdots & \vdots & \ddots & \vdots \\
0 & 0 & \ldots & A_{n}
\end{array}\right)
$$

est également multiplicative.

Remarque (3.2.3). L'espace quantique sous-jacent à un monoïde quantique matriciel est de type fini. On dira qu'un tel monoïde quantique est de type fini. On démontre que si $K$ est un corps alors un monoïde quantique est matriciel si et seulement s'il est de type fini.

\subsection{Familles de morphismes de cônes quantiques}

Définition (3.3.1). Soient $C$ et $C^{\prime}$ deux cônes quantiques et $X$ un espace quantique. On appelle famille de morphismes de cônes quantiques de $C$ dans $C^{\prime}$ indexée par 
l'espace quantique $X$ (ou plus simplement famille de morphismes de $C$ dans $C^{\prime}$ indexée par $X$ ) un morphisme d'espaces quantiques $f: X \times C \rightarrow C^{\prime}$ (où l'on désigne aussi par $C$ (resp. $C^{\prime}$ ) l'espace quantique sous-jacent au cône quantique $C$ (resp. $C^{\prime}$ )) tel que

$$
f^{*}\left(\Omega_{C^{\prime}}^{1,0}\right) \subset \Omega_{X} \otimes \Omega_{C}^{1,0} .
$$

Si $f^{\prime}: X^{\prime} \times C \rightarrow C^{\prime}$ désigne une famille de morphismes de $C$ dans $C^{\prime}$ indexée par l'espace quantique $X^{\prime}$, on appelle morphisme de $f$ dans $f^{\prime}$ un morphisme d'espaces quantiques $g: X \rightarrow X^{\prime}$ tel que le diagramme

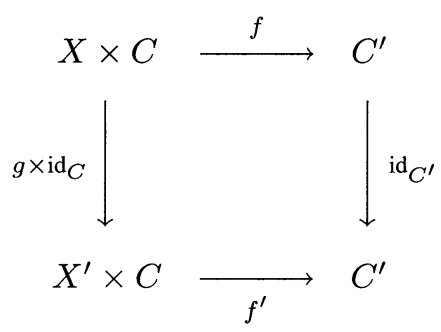

soit commutatif. On définit ainsi la catégorie des familles de morphismes de cônes quantiques de $C$ dans $C^{\prime}$.

Théorème (3.3.2). Soient $C$ et $C^{\prime}$ deux cônes quantiques. On suppose que

i) $K$ est un corps;

ii) $C$ et $C^{\prime}$ sont de type fini.

Alors la catégorie des familles de morphismes de $C$ dans $C^{\prime}$ possède un objet final. Autrement dit, il existe un espace quantique $X_{0}$ et une famille de morphismes de $C$ dans $C^{\prime}$ indexée par $X_{0}$

$$
f_{0}: X_{0} \times C \rightarrow C^{\prime}
$$

tels que pour toute famille de morphismes de $C$ dans $C^{\prime}$ indexée par un espace quantique $X$

$$
f: X \times C \rightarrow C^{\prime}
$$

il existe un morphisme unique d'espaces quantiques $g: X \rightarrow X_{0}$ tel que le diagramme

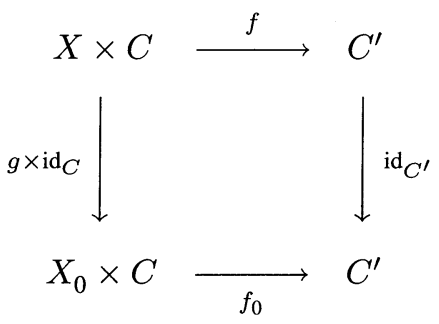

soit commutatif.

Démonstration. Il résulte de l'hypothèse (ii) que $\Omega_{C}^{1,0}$ et $\Omega_{C^{\prime}}^{1,0}$ sont des $K$-espaces vectoriels de dimension finie. Soient $b_{1}, \ldots, b_{n}$ (resp. $b_{1}^{\prime}, \ldots, b_{n^{\prime}}^{\prime}$ ) une base de $\Omega_{C}^{1,0}$ (resp. $\left.\Omega_{C^{\prime}}^{1,0}\right), \Omega=K\left\langle\left\langle x_{1}, \ldots, x_{n}\right\rangle\right\rangle$ (resp. $\Omega^{\prime}=K\left\langle\left\langle x_{1}^{\prime}, \ldots, x_{n^{\prime}}^{\prime}\right\rangle\right\rangle$ ) l'algèbre différentielle libre engendrée par la famille des indéterminées $\left(x_{j}\right)_{1 \leq j \leq n}$ (resp. $\left.\left(x_{j^{\prime}}^{\prime}\right)_{1 \leq j^{\prime} \leq n^{\prime}}\right), \varphi: \Omega \rightarrow \Omega_{C}$ (resp. $\varphi^{\prime}: \Omega^{\prime} \rightarrow \Omega_{C^{\prime}}$ ) le morphisme (surjectif) d'algèbres 
différentielles défini par $\varphi\left(x_{j}\right)=b_{j}$ (resp. $\varphi^{\prime}\left(x_{j^{\prime}}^{\prime}\right)=b_{j^{\prime}}^{\prime}$ ), pour $1 \leq j \leq n$ (resp. pour $1 \leq j^{\prime} \leq n^{\prime}$ ) et $J$ (resp. $J^{\prime}$ ) le noyau de $\varphi$ (resp. $\varphi^{\prime}$ ) qui est un idéal différentiel bigradué de $\Omega$ (resp. $\Omega^{\prime}$ ) (cf. 2.4.2). Comme $K$ est un corps, il existe un sous-espace vectoriel bigradué $M$ de $\Omega$ tel que $\Omega=J \oplus M$ et une base $\left(s_{i}\right)_{i \in I}\left(\operatorname{resp} .\left(s_{i^{\prime}}^{\prime}\right)_{i^{\prime} \in I^{\prime}}\right)$ de $M$ (resp. de $J$ ) formée d'éléments bihomogènes. Enfin, soit $\left(t_{l}\right)_{l \in L}$ un système de générateurs bihomogènes de $J^{\prime}$ en tant qu'idéal différentiel. On désigne par $H$ l'algèbre différentielle libre $K\left\langle\left\langle\left(a_{j^{\prime}}^{j}\right)_{1 \leq j \leq n, 1 \leq \jmath^{\prime} \leq n^{\prime}}\right\rangle\right\rangle$ engendrée par la famille des indéterminées $\left(a_{j^{\prime}}^{j}\right)_{1 \leq j \leq n, 1 \leq j^{\prime} \leq n^{\prime}}$ et on définit un morphisme d'algèbres différentielles

$$
\psi: \Omega^{\prime} \rightarrow H^{g} \otimes \Omega
$$

par $\psi\left(x_{j^{\prime}}\right)=\sum_{j=1}^{n} a_{j^{\prime}}^{j} \otimes x_{j}$, pour $1 \leq j^{\prime} \leq n^{\prime}$. Pour tout $l, l \in L$,

$$
\psi\left(t_{l}\right)=\sum_{i \in I} r_{l i} \otimes s_{i}+\sum_{i^{\prime} \in I^{\prime}} r_{l i^{\prime}}^{\prime} \otimes s_{i^{\prime}}^{\prime}
$$

où $r_{l i}, r_{l i^{\prime}}^{\prime} \in H$, les familles $\left(r_{l i}\right)_{l \in L, i \in I}$ et $\left(r_{l i^{\prime}}^{\prime}\right)_{l \in L, i^{\prime} \in I^{\prime}}$ étant uniquement déterminées par ces formules. Soit $R$ l'idéal différentiel de $H$ engendré par $\left(r_{l \downarrow}\right)_{l \in L, i \in I}$. Le morphisme $\psi$ définit par passage au quotient un morphisme d'algèbres différentielles

$$
\bar{\psi}: \Omega_{C^{\prime}} \rightarrow H / R^{g} \otimes \Omega_{C}
$$

(en identifiant $\Omega_{C}$ à $\Omega / J$ et $\Omega_{C^{\prime}}$ à $\left.\Omega^{\prime} / J^{\prime}\right)$. Si l'on pose $X_{0}=\operatorname{Spec}(H / R)$ et $f_{0}=\operatorname{Spec}(\bar{\psi})$, alors

$$
f_{0}: X_{0} \times C \rightarrow C^{\prime}
$$

est une famille de morphismes de $C$ dans $C^{\prime}$ indexée par $X_{0}$. Démontrons qu'elle satisfait à la propriété universelle du théorème. Soit $f: X \times C \rightarrow C^{\prime}$ une famille de morphismes de $C$ dans $C^{\prime}$ indexée par $X$. Comme

$$
f^{*}\left(\Omega_{C^{\prime}}^{1,0}\right) \subset \Omega_{X} \otimes \Omega_{C}^{1,0},
$$

pour tout $j^{\prime}, 1 \leq j^{\prime} \leq n^{\prime}$, il existe une famille unique $\left(b_{j^{\prime}}^{j}\right)_{1 \leq j \leq n}$ d'éléments de $\Omega_{X}^{0}$ telle que

$$
f^{*}\left(b_{j^{\prime}}\right)=\sum_{j=1}^{n} b_{j^{\prime}}^{j} \otimes b_{j}
$$

(car $\left(b_{j}\right)_{1 \leq j \leq n}$ est une base de $\Omega_{C}^{1,0}$ ). On en déduit un morphisme unique d'algèbres différentielles

$$
\chi: H \rightarrow \Omega_{X}
$$

tel que pour tout $j$ et $j^{\prime}, 1 \leq j \leq n, 1 \leq j^{\prime} \leq n^{\prime}, \chi\left(a_{j^{\prime}}^{j}\right)=b_{j^{\prime}}^{j}$, ou ce qui est équivalent, rendant commutatif le diagramme suivant

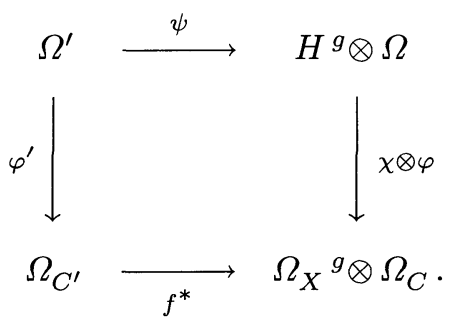


Pour tout $l, l \in L$, on a

$$
(\chi \otimes \varphi) \circ \psi\left(t_{l}\right)=f^{*} \circ \varphi^{\prime}\left(t_{l}\right)=0
$$

d'où

$$
\sum_{i \in I} \chi\left(r_{l i}\right) \otimes \varphi\left(s_{i}\right)=0 .
$$

La famille $\left(s_{i}\right)_{i \in I}$ étant une base de $M$, la famille $\left(\varphi\left(s_{i}\right)\right)_{i \in I}$ est une base du $K$-espace vectoriel $\Omega_{C}$, on a donc pour tout $i, i \in I, \chi\left(r_{l i}\right)=0$. On en déduit que $\chi$ induit un morphisme d'algèbres différentielles

$$
\bar{\chi}: H / R \rightarrow \Omega_{X}
$$

tel que si l'on pose $g=\operatorname{Spec}(\bar{\chi})$, le diagramme

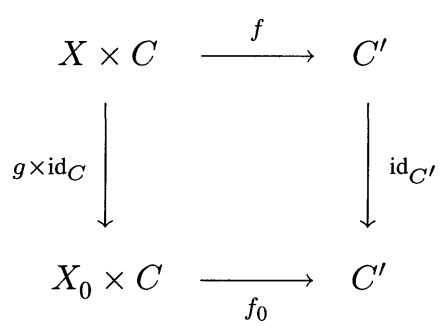

soit commutatif, ce qui démontre le théorème.

(3.3.3). On dira que la famille de morphismes $f_{0}: X_{o} \times C \rightarrow C^{\prime}$ satisfaisant aux propriétés du théorème (3.3.2) est la famille universelle de morphismes de cônes quantiques de $C$ dans $C^{\prime}$. La démonstration de ce théorème en donne une construction explicite, très utile pour étudier des exemples. On vérifie facilement que $X_{0}$ peut être canoniquement muni d'une structure de cône quantique, mais $f_{0}$ n'est pas un morphisme de cônes quantiques pour cette structure.

\subsection{Action d'un monoïde quantique sur un espace quantique}

Définition (3.4.1). Soient $(M, m, e)$ un monoïde quantique et $X$ un espace quantique. On appelle action (à gauche) de $M$ sur $X$ un morphisme d'espaces quantiques $f: M \times X \rightarrow X$ tel que les diagrammes suivants soient commutatifs:

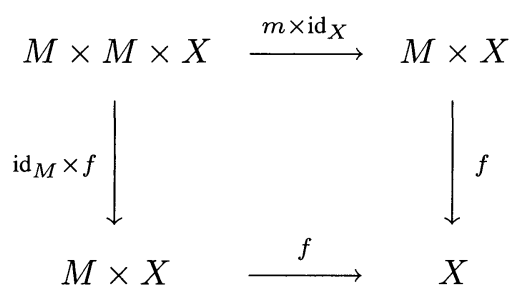

et

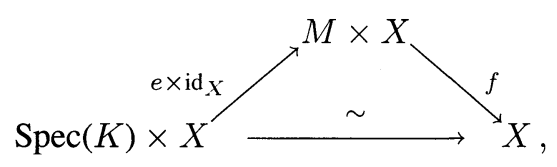


l'isomorphisme $\operatorname{Spec}(K) \times X \stackrel{\sim}{\rightarrow} X$ étant la contrainte d'unité [cf. (2.3.7)]. Si $C$ désigne un cône quantique, on appelle action de $M$ sur $C$ un morphisme d'espaces quantiques $f: M \times C \rightarrow C$ qui est à la fois une action de $M$ sur l'espace quantique sous-jacent à $C$ et une famille de morphismes de cônes quantiques de $C$ dans $C$. On dit alors que le monoïde quantique $M$ agit sur l'espace quantique $X$, ou sur le cône quantique $C$.

Théorème (3.4.2). Supposons que $K$ soit un corps et soient $C$ un cône quantique de type fini et $f_{0}: X_{0} \times C \rightarrow C$ la famille universelle de morphismes de cônes quantiques de $C$ dans lui-même. Alors il existe une structure unique de monoïde quantique sur $X_{0}$ telle que $f_{0}$ soit une action de $X_{0}$ sur $C$. De plus, pour cette structure $X_{0}$ est un monoïde quantique matriciel.

Démonstration. Le morphisme composé

$$
f_{0} \circ\left(\operatorname{id}_{X_{0}} \times f_{0}\right): X_{0} \times X_{0} \times C \stackrel{\mathrm{id}_{X_{0}} \times f_{0}}{\longrightarrow} X_{0} \times C \stackrel{f_{0}}{\longrightarrow} C
$$

(resp. la contrainte d'unité [cf. (2.3.7.1)]

$$
\left.\lambda_{C}: \operatorname{Spec}(K) \times C \rightarrow C\right)
$$

est une famille de morphismes de $C$ dans lui-même indexée par $X_{0} \times X_{0}$ (resp. par $\operatorname{Spec}(K))$ et il résulte de la propriété universelle de $f_{0}$ (3.3.2) qu'il existe un morphisme d'espaces quantiques $m: X_{0} \times X_{0} \rightarrow X_{0}$ (resp. $e: \operatorname{Spec}(K) \rightarrow X_{0}$ ) tel que

$$
f_{0} \circ\left(m \times \mathrm{id}_{C}\right)=f_{0} \circ\left(\mathrm{id}_{X_{0}} \times f_{0}\right)
$$

(resp.

$$
\left.f_{0} \circ\left(e \times \mathrm{id}_{C}\right)=\lambda_{C}\right)
$$

Il résulte aussitôt de la partie unicité de la propriété universelle que $\left(X_{0}, m, e\right)$ est un monoïde quantique et des égalités (3.4.2.1) et (3.4.2.2) que $f_{0}$ est une action de ce monoïde sur $C$. L'unicité de la structure de monoïde quantique sur $X_{0}$ résulte de l'unicité de $m$ et $e$ satisfaisant à (3.4.2.1) et (3.4.2.2) [cf. (3.3.2)]. Le fait que le monoïde quantique $\left(X_{0}, m, e\right)$ est matriciel résulte de la construction explicite de $X_{0}$, décrite dans la démonstration du théorème (3.3.2). Les détails des vérifications sont laissés au lecteur. On peut d'ailleurs obtenir ainsi une démonstration directe du fait que $\left(X_{0}, m, e\right)$ est un monoïde quantique.

(3.4.3). On dira que $\left(X_{0}, m, e\right)$ est le monoïde quantique des endomorphismes du cône quantique $C$ et que $f_{0}: X_{0} \times C \rightarrow C$ est $l$ 'action canonique de ce monoïde sur $C$. La démonstration ci-dessus, qui est une adaptation de celle de Manin [Man2], a le mérite d'être valable dans n'importe quelle catégorie monoïdale, pourvu qu'on ait un théorème analogue au théorème (3.3.2). Le monoïde quantique $X_{0}$ des endomorphismes d'un cône quantique $C$, muni de l'action canonique $f_{0}: X_{0} \times C \rightarrow C$, satisfait à la propriété universelle suivante: pour tout monoïde quantique $X$ et toute action $f: X \times C \rightarrow C$ de $X$ sur le cône quantique $C$, il existe un morphisme unique de monoïdes quantiques $g: X \rightarrow X_{0}$ tel que $f=f_{0}\left(g \times \mathrm{id}_{C}\right)$. 


\subsection{Exemples de monoïdes quantiques}

Notations (3.5.1). Soient $n$ un entier, $n \in \mathbb{N}, R=\left(r_{i i^{\prime}}^{j j^{\prime}}\right)_{1 \leq i, i^{\prime}, j, j^{\prime} \leq n}$ une matrice carrée $n^{2} \times n^{2}$, à coefficients dans $K$ et $U$ et $V$ des relations binaires définies sur l'ensemble $\{1, \ldots, n\}$. En pratique

$$
U, V \in\{=, \leq,<, \geq,>\} \text {. }
$$

On pose

$$
\begin{aligned}
& R_{U}^{V}=\left(r_{i i^{\prime}}^{j j^{\prime}}\right)_{1 \leq i, i^{\prime}, j, j^{\prime} \leq n, i U i^{\prime}, j V j^{\prime}} \\
& R_{U}=\left(r_{i i^{\prime}}^{j j^{\prime}}\right)_{1 \leq i, i^{\prime}, j, j^{\prime} \leq n, i U i^{\prime}}, \\
& R^{V}=\left(r_{i i^{\prime}}^{j j^{\prime}}\right)_{1 \leq i, i^{\prime}, j, j^{\prime} \leq n, j V j^{\prime}}
\end{aligned}
$$

Si $S$ désigne une matrice du même type, $W$ une autre relation et $\bar{W}$ sa négation,

$$
i \bar{W} i^{\prime} \Leftrightarrow \text { non } i W i^{\prime}
$$

on a

$$
\begin{aligned}
(R S)_{U}^{V} & =R_{U}^{W} S_{W}^{V}+R_{U}^{\bar{W}} S_{\bar{W}}^{V} \\
(R S)_{U} & =R_{U}^{W} S_{W}+R_{U}^{\bar{W}} S_{\bar{W}} \\
(R S)^{V} & =R^{W} S_{W}^{V}+R^{\bar{W}} S_{\bar{W}}^{V} \\
R S & =R^{W} S_{W}+R^{\bar{W}} S_{\bar{W}}
\end{aligned}
$$

par exemple,

$$
(R S)_{\geq}^{\leq}=R_{\geq}^{<} S_{<}^{\leq}+R_{\geq}^{\geq} S_{\geq}^{\leq}
$$

Si $I$ désigne la matrice unité,

$$
I=\left(\delta_{i i^{\prime}}^{j j^{\prime}}\right)_{1 \leq i, i^{\prime}, j, j^{\prime} \leq n}
$$

on remarque que $I_{W}^{W}$ est encore une matrice unité, tandis que $I_{W}^{\bar{W}}=0$. Par exemple,

$$
I_{\leq}^{>}=0, \quad I_{<}^{\geq}=0 \quad \text { etc. }
$$

Si $m, m^{\prime}, n$ et $n^{\prime}$ désignent des entiers, $m, m^{\prime}, n, n^{\prime} \in \mathbb{N}$ et $R=\left(r_{i}^{j}\right)_{1 \leq i \leq m, 1 \leq j \leq n}$ et $S=\left(s_{i^{\prime}}^{j^{\prime}}\right)_{1 \leq i^{\prime} \leq m^{\prime}, 1 \leq j^{\prime} \leq n^{\prime}}$ des matrices à coefficients dans $K$, on désigne par $R \otimes S$ la matrice

$$
R \otimes S=\left(r_{i}^{j} s_{i^{\prime}}^{j^{\prime}}\right)_{1 \leq i \leq m, 1 \leq i^{\prime} \leq m^{\prime}, 1 \leq j \leq n, 1 \leq j^{\prime} \leq n^{\prime}}
$$

produit tensoriel des matrices $R$ et $S$.

Exemple (3.5.2). On suppose que $K$ soit un corps. Soient $n$ un entier, $n \in \mathbb{N}$,

$$
\begin{aligned}
& Q=\left(q_{i i^{\prime}}^{j j^{\prime}}\right)_{1 \leq i^{\prime}<\imath \leq n, 1 \leq j \leq j^{\prime} \leq n}, \\
& R=\left(r_{i i^{\prime}}^{j j^{\prime}}\right)_{1 \leq i, i^{\prime}, j, j^{\prime} \leq n}, \\
& P=\left(p_{i i^{\prime}}^{j j^{\prime}}\right)_{1 \leq i \leq i^{\prime} \leq n, 1 \leq j^{\prime}<j \leq n},
\end{aligned}
$$

des matrices à coefficients dans $K, \Omega_{(Q, R, P)}=\Omega / J$ l'algèbre différentielle graduée quotient de l'algèbre différentielle libre $\Omega=K\left\langle\left\langle x_{1}, \ldots, x_{n}\right\rangle\right\rangle$, engendrée par la 
famille des indéterminées $\left(x_{i}\right)_{1 \leq i \leq n}$, par l'idéal différentiel $J$ engendré par les relations

$$
\begin{aligned}
x_{i} x_{i^{\prime}}=\sum_{j \leq j^{\prime}} q_{i i^{\prime}}^{j j^{\prime}} x_{j} x_{j^{\prime}}, & 1 \leq i^{\prime}<i \leq n, \\
\xi_{i} x_{i^{\prime}}=\sum_{j, j^{\prime}} r_{i i^{\prime}}^{j j^{\prime}} x_{j} \xi_{j^{\prime}}, & 1 \leq i, i^{\prime} \leq n, \\
\xi_{i} \xi_{i^{\prime}}=\sum_{j^{\prime}<j} p_{i i^{\prime}}^{j j^{\prime}} \xi_{j} \xi_{j^{\prime}}, & 1 \leq i \leq i^{\prime} \leq n,
\end{aligned}
$$

où $\xi_{i}=d x_{i}$, et $\mathbb{A}_{(Q, R, P)}^{n}=\operatorname{Spec}\left(Q_{(Q, R, P)}\right)$ l'espace quantique correspondant (espace affine quantique de dimension $n$ associé aux matrices $Q, R$ et $P$ ). L'idéal $J$ est bigradué et l'espace $\mathbb{A}_{(Q, R, P)}^{n}$ est ainsi muni d'une structure de cône quantique. En utilisant les notations de (3.5.1), les relations définissant l'idéal $J$ s'écrivent de façon plus compacte:

$$
\begin{aligned}
(x \otimes x)_{>} & =Q_{>}^{\leq}(x \otimes x)_{\leq}, \\
\xi \otimes x & =R(x \otimes \xi), \\
(\xi \otimes \xi)_{\leq} & =P_{\leq}^{>}(\xi \otimes \xi)_{>},
\end{aligned}
$$

où $x$ désigne la matrice $\left(x_{i}\right)_{1 \leq i \leq n}$, $\xi$ la matrice $\left(\xi_{i}\right)_{1 \leq i \leq n}$ et $Q_{>}^{\leq}=Q$ et $P_{\leq}^{>}=P$, cette notation étant purement mnémotechnique, destinée à rappeler la forme des matrices $Q$ et $R$ (mais compatible avec les notations de (3.5.1), si l'on complète ces matrices de façon arbitraire en des matrices $n^{2} \times n^{2}$ ).

On remarque que

$$
\left(x_{i_{1}} \ldots x_{i_{m}} \xi_{j_{1}} \ldots \xi_{j_{m^{\prime}}}\right)_{1 \leq i_{1} \leq \ldots \leq i_{m} \leq n, 1 \leq j_{m^{\prime}}<\ldots<j_{1} \leq n}
$$

est un système de générateurs du $K$-espace vectoriel $\Omega_{(Q, R, P)}$. On s'intéresse plus particulièrement au cas où le système ci-dessus est une base, autrement dit au cas où la fonction de Hilbert de $\Omega_{(Q, R, P)}$ est la même que celle du complexe de de Rham algébrique habituel sur l'anneau des polynômes commutatifs à $n$ indéterminées:

$$
\operatorname{dim}_{K}\left(\Omega_{(Q, R, P)}^{m, m^{\prime}}\right)=\left(\begin{array}{c}
m+n-1 \\
n-1
\end{array}\right) \cdot\left(\begin{array}{c}
n \\
m^{\prime}
\end{array}\right) .
$$

Supposons qu'il en soit ainsi, ou du moins que

$$
\left(x_{i} x_{j}\right)_{1 \leq i \leq j \leq n}, \quad\left(x_{i} \xi_{j}\right)_{1 \leq i, j \leq n}, \quad\left(\xi_{j} \xi_{i}\right)_{1 \leq i \leq j \leq n}
$$

soit une famille libre. En différentiant la relation (3.5.2.1), on obtient

$$
(\xi \otimes x)_{>}+(x \otimes \xi)_{>}=Q_{>}^{\leq}(\xi \otimes x)_{\leq}+Q_{>}^{\leq}(x \otimes \xi)_{\leq},
$$

ce qui implique en utilisant (3.5.2.2) que

$$
R_{>}(x \otimes \xi)+I_{>}(x \otimes \xi)=Q_{>}^{\leq} R_{\leq}(x \otimes \xi)+Q_{>}^{\leq} I_{\leq}(x \otimes \xi),
$$

où $I$ désigne la matrice unité $n^{2} \times n^{2}$, et en vertu de l'hypothèse, on a

$$
R_{>}+I_{>}=Q_{>}^{\leq}\left(R_{\leq}+I_{\leq}\right) .
$$

Cette relation se décompose en deux autres

$$
R_{>}^{\leq}=Q_{>}^{\leq}\left(R_{\leq}^{\leq}+I_{\leq}^{\leq}\right)
$$


et

$$
R_{>}^{>}+I_{>}^{>}=Q_{>}^{\leq} R_{\leq}^{>}
$$

Supposons que

$$
\operatorname{rg}_{K}(R+I)=\operatorname{rg}_{K}\left(R_{\leq}^{\leq}+I_{\leq}^{\leq}\right)=n(n+1) / 2 .
$$

Alors $R_{\leq}^{\leq}+I_{\leq}$est inversible,

$$
Q_{>}^{\leq}=R_{>}^{\leq}\left(R_{\leq}^{\leq}+I_{\leq}^{\leq}\right)^{-1}
$$

et cette égalité implique (3.5.2.4).

De même, en différentiant (3.5.2.2), on obtient

$$
-(\xi \otimes \xi)=R(\xi \otimes \xi),
$$

ce qui implique

$$
\left(R^{\leq}+I^{\leq}\right)(\xi \otimes \xi)_{\leq}+\left(R^{>}+I^{>}\right)(\xi \otimes \xi)_{>}=0,
$$

d'où, en utilisant (3.5.2.3)

$$
\left(R^{\leq}+I^{\leq}\right) P_{\leq}^{>}(\xi \otimes \xi)_{>}+\left(R^{>}+I^{>}\right)(\xi \otimes \xi)_{>}=0,
$$

et comme la famille $\left(\xi_{j} \xi_{i}\right)_{1 \leq i<j \leq n}$ est supposée libre, on a

$$
\left(R^{\leq}+I^{\leq}\right) P_{\leq}^{>}+R^{>}+I^{>}=0 .
$$

La relation ci-dessus se décompose en deux:

$$
\left(R_{\leq}^{\leq}+I_{\leq}^{\leq}\right) P_{\leq}^{>}+R_{\leq}^{>}=0
$$

et

$$
R_{>}^{\leq} P_{\leq}^{>}+R_{>}^{>}+I_{>}^{>}=0 .
$$

On a donc sous l'hypothèse (3.5.2.7)

$$
P_{\leq}^{>}=-\left(R_{\leq}^{\leq}+I_{\leq}^{\leq}\right)^{-1} R_{\leq}^{>},
$$

relation qui implique alors (3.5.2.9).

Réciproquement, soit $R$ une matrice carrée $n^{2} \times n^{2}$ satisfaisant à l'hypothèse (3.5.2.7), supposons que les matrices $Q \lesseqgtr$ et $P_{<}^{>}$soient définies par les formules (3.5.2.8) et (3.5.2.12) et l'idéal $J$ par les relations (3.5.2.1), (3.5.2.2) et (3.5.2.3). Alors on pose $\Omega_{R}=\Omega_{(Q, R, P)}$ et $\mathbb{A}_{R}^{n}=\mathbb{A}_{(Q, R, P)}^{n}$ et en vertu de ce qui précède, on vérifie facilement que $J$ est l'idéal différentiel engendré par les relations (3.5.2.1) et (3.5.2.2) et que $\left(x_{i} x_{j}\right)_{1 \leq i \leq j \leq n}$ est une base de $\Omega_{R}^{2,0},\left(x_{i} \xi_{j}\right)_{1 \leq i, j \leq n}$ une base de $\Omega_{R}^{1,1}$ et $\left(\xi_{j} \xi_{i}\right)_{1 \leq i<j \leq n}$ une base de $\Omega_{R}^{0,2}$.

En complétant donc la réunion de ces trois familles en une base de $\Omega_{R}$, on peut appliquer la méthode du théorème (3.3.2) pour construire le monoïde quantique des endomorphismes du cône quantique $\mathbb{A}_{R}^{n}$ [cf. (3.4.3)] (monoïde quantique des matrices $n \times n$, associé à la matrice $R$ ). On démontre facilement la proposition suivante:

Proposition (3.5.3). Sous ces hypothèses, le monoïde quantique des endomorphismes du cône quantique $\mathbb{A}_{R}^{n}$ est isomorphe à

$$
\operatorname{Spec}(H / J(R)),
$$


où

$$
H=K\left\langle\left\langle\left(a_{i}^{j}\right)_{1 \leq i, j \leq n}\right\rangle\right\rangle
$$

désigne l'algèbre différentielle libre engendrée par la famille des indéterminées $\left(a_{i}^{j}\right)_{1 \leq i, j \leq n}$ et $J(R)$ est l'déal différentiel de $H$ engendré par les coefficients des matrices

$$
(A \otimes A) R-R(A \otimes A)
$$

et

$$
[d A \otimes A-R(A \otimes d A)](R+I) \leq,
$$

où $A=\left(a_{i}^{j}\right)_{1 \leq i, j \leq n}$ et $d A=\left(d a_{i}^{j}\right)_{1 \leq i, j \leq n}$.

Exemple (3.5.4). (Calcul différentiel sur la déformation multiparamétrique de l'algèbre des fonctions sur les matrices, introduite par M. Artin, W. Schelter, J. Tate [AST]). On suppose toujours que $K$ soit un corps. Soient $n$ un entier, $n \in \mathbb{N}$, et $q=\left(q_{j i}\right)_{1 \leq i<j \leq n}, p=\left(p_{i j}\right)_{1 \leq i<j \leq n}$ et $\lambda=\left(\lambda_{j}\right)_{1 \leq j \leq n}$ des familles d'éléments de $K^{*}$ satisfaisant à la relation

$$
\lambda_{j}=q_{j i} p_{i j}, \quad 1 \leq i<j \leq n .
$$

En gardant les notations de l'exemple (3.5.2), on définit des matrices $Q=Q_{>}^{\leq}, R$ et $P=P_{\leq}^{>}$par

$$
\begin{array}{ll}
q_{j i}^{i j}=q_{j i}, & 1 \leq i<j \leq n, \\
r_{i i}^{i i}=\lambda_{i}, & 1 \leq i \leq n, \\
r_{i j}^{j i}=p_{i j}, & 1 \leq i<j \leq n, \\
r_{j i}^{i j}=q_{j i}, & 1 \leq i<j \leq n, \\
r_{j i}^{j i}=\lambda_{j}-1, & 1 \leq i<j \leq n, \\
p_{i j}^{j i}=-p_{i j}, & 1 \leq i<j \leq n,
\end{array}
$$

tous les autres coefficients étant nuls. On vérifie facilement que les matrices $Q, R, P$ satisfont aux conditions (3.5.2.4) et (3.5.2.9) et que

$$
\left(x_{i_{1}} \ldots x_{i_{m}} \xi_{j_{1}} \ldots \xi_{j_{m^{\prime}}}\right)_{1 \leq i_{1} \leq \ldots \leq i_{m} \leq n, 1 \leq j_{m^{\prime}}<\ldots<j_{1} \leq n}
$$

est une base de l'algèbre différentielle $\Omega_{(Q, R, P)}$. La condition (3.5.2.7) est équivalente à

$$
\lambda_{j} \neq-1, \quad 1 \leq j \leq n,
$$

et l'espace quantique $\mathbb{A}_{q, p, \lambda}^{n}=\mathbb{A}_{(Q, R, P)}^{n}$ est simple, si et seulement si cette condition est satisfaite. Soit $\mathbb{M}_{q, p, \lambda}^{n}$ le monoïde quantique des endomorphismes du cône quantique $\mathbb{A}_{q, p, \lambda}^{n}$. L'espace quantique $\mathbb{M}_{q, p, \lambda}^{n}$ est muni canoniquement d'une structure de cône quantique [cf. (3.3.3)], mais on constate (en utilisant les formules de la proposition (3.5.3)) que la fonction de Hilbert de son complexe de de Rham $\Omega_{q, p, \lambda}$ n'est pas la même que celle du complexe de de Rham algèbrique de l'anneau des polynômes commutatifs sur l'espace affine des matrices carrées $n \times n$, autrement dit, que

$$
\operatorname{dim}_{K}\left(\Omega_{q, p, \lambda}^{m, m^{\prime}}\right) \neq\left(\begin{array}{c}
m+n^{2}-1 \\
n^{2}-1
\end{array}\right) \cdot\left(\begin{array}{c}
n^{2} \\
m^{\prime}
\end{array}\right) .
$$

Néanmoins, on démontre le théorème suivant [Ma12]. 
Théorème (3.5.5). Pour qu'il existe un monoïde quantique $\mathbb{M}$ agissant sur le cône quantique $\mathbb{A}_{q, p, \lambda}^{n}$, tel que

$$
\operatorname{dim}_{K}\left(\Omega_{\mathbb{M}}^{m, m^{\prime}}\right)=\left(\begin{array}{c}
m+n^{2}-1 \\
n^{2}-1
\end{array}\right) \cdot\left(\begin{array}{l}
n^{2} \\
m^{\prime}
\end{array}\right)
$$

et tel que si $g: \mathbb{M} \rightarrow \mathbb{M}_{q, p, \lambda}^{n}$ désigne le morphisme de monoïdes quantiques défini par la propriété universelle de $\mathbb{M}_{q, p, \lambda}^{n}$ [cf. (3.4.3)], $g^{*}: \Omega_{q, p, \lambda} \rightarrow \Omega_{\mathbb{M}}$ soit surjectif, il faut et il suffit que pour tout $i$ et $j, 1 \leq i, j \leq n$, on ait $\lambda_{i}=\lambda_{j}$, et alors ce monoïde quantique $\mathbb{M}$ est unique, à isomorphisme près, et l'algèbre différentielle graduée $\Omega_{\mathbb{M}}$ est isomorphe au quotient de l'algèbre différentielle libre engendrée par les indéterminées $\left(a_{i}^{j}\right)_{1 \leq i, j \leq n}$ par l'idéal bilatère engendré par

(1) $a_{j}^{k} a_{i}^{k}-q_{j i} a_{i}^{k} a_{j}^{k}$,

$1 \leq i<j \leq n, 1 \leq k \leq n$

(2) $a_{i}^{l} a_{i}^{k}-p_{k l} a_{i}^{k} a_{i}^{l}$,

$1 \leq i \leq n, 1 \leq k<l \leq n$

(3) $a_{j}^{k} a_{i}^{l}-\left(p_{i j}\right)^{-1} q_{l k} a_{i}^{l} a_{\jmath}^{k}$,

$1 \leq i<j \leq n, 1 \leq k<l \leq n$

(4) $a_{j}^{l} a_{i}^{k}-\left(p_{i j}\right)^{-1} p_{k l} a_{i}^{k} a_{j}^{l}-\left(p_{i j}\right)^{-1}(\lambda-1) a_{i}^{l} a_{j}^{k}$

(5) $\alpha_{i}^{k} a_{i}^{k}-\lambda a_{i}^{k} \alpha_{i}^{k}$,

$1 \leq i<j \leq n, 1 \leq k<l \leq n$

(6) $\alpha_{i}^{k} a_{j}^{k}-p_{i j} a_{\jmath}^{k} \alpha_{i}^{k}$,

$1 \leq i \leq n, 1 \leq k \leq n$

(7) $\alpha_{j}^{k} a_{i}^{k}-q_{j \imath} a_{\imath}^{k} \alpha_{j}^{k}-(\lambda-1) a_{j}^{k} \alpha_{i}^{k}$,

$1 \leq i<j \leq n, 1 \leq k \leq n$

(8) $\alpha_{i}^{k} a_{i}^{l}-q_{l k} a_{i}^{l} \alpha_{\imath}^{k}$,

$1 \leq i<j \leq n, 1 \leq k \leq n$

(9) $\alpha_{i}^{l} a_{i}^{k}-p_{k l} a_{i}^{k} \alpha_{i}^{l}-(\lambda-1) a_{i}^{l} \alpha_{i}^{k}$,

$1 \leq i \leq n, 1 \leq k<l \leq n$

(10) $\alpha_{i}^{k} a_{j}^{l}-p_{i j}\left(p_{k l}\right)^{-1} a_{j}^{l} \alpha_{i}^{k}$,

$1 \leq i \leq n, 1 \leq k<l \leq n$

(11) $\alpha_{i}^{l} a_{j}^{k}-\left(q_{j i}\right)^{-1} p_{k l} a_{j}^{k} \alpha_{i}^{l}-\left(q_{j i}\right)^{-1}(\lambda-1) a_{\jmath}^{l} \alpha_{i}^{k}, \quad 1 \leq i<j \leq n, 1 \leq k<l \leq n$

(12) $\alpha_{j}^{k} a_{i}^{l}-q_{j i}\left(p_{k l}\right)^{-1} a_{i}^{l} \alpha_{j}^{k}-\left(p_{k l}\right)^{-1}(\lambda-1) a_{j}^{l} \alpha_{i}^{k}, \quad 1 \leq i<j \leq n, 1 \leq k<l \leq n$

(13) $\alpha_{j}^{l} a_{i}^{k}-q_{j i}\left(q_{l k}\right)^{-1} a_{i}^{k} \alpha_{j}^{l}-\left(p_{i j}\right)^{-1}(\lambda-1) a_{i}^{l} \alpha_{j}^{k}-\left(q_{l k}\right)^{-1}(\lambda-1) a_{j}^{k} \alpha_{i}^{l}$

$-\left(\lambda-2+\lambda^{-1}\right) a_{j}^{l} \alpha_{i}^{k}, \quad 1 \leq i<j \leq n, 1 \leq k<l \leq n$

(14) $\left(\alpha_{i}^{k}\right)^{2}$,

$1 \leq i \leq n, 1 \leq k \leq n$

(15) $\alpha_{i}^{k} \alpha_{j}^{k}+p_{\imath j} \alpha_{j}^{k} \alpha_{i}^{k}$

$1 \leq i<j \leq n, 1 \leq k \leq n$

(16) $\alpha_{i}^{k} \alpha_{i}^{l}+q_{l k} \alpha_{i}^{l} \alpha_{i}^{k}$,

$1 \leq i \leq n, 1 \leq k<l \leq n$

(17) $\alpha_{i}^{k} \alpha_{j}^{l}+p_{i j}\left(p_{k l}\right)^{-1} \alpha_{\jmath}^{l} \alpha_{\imath}^{k}$,

$1 \leq i<j \leq n, 1 \leq k<l \leq n$

(18) $\alpha_{i}^{l} \alpha_{j}^{k}+\left(q_{j i}\right)^{-1} p_{k l} \alpha_{j}^{k} \alpha_{i}^{l}+\left(q_{j i}\right)^{-1}(\lambda-1) \alpha_{j}^{l} \alpha_{i}^{k}, 1 \leq i<j \leq n, 1 \leq k<l \leq n$ où $\alpha_{i}^{j}=d a_{i}^{j}$ et $\lambda=\lambda_{1}=\ldots=\lambda_{n}$.

\section{Groupes quantiques}

\subsection{Groupes quantiques}

Définition (4.1.1). Soit $(X, m, e)$ un monoïde quantique. On dit qu'un morphisme d'espaces quantiques $i: X \rightarrow X^{\text {opp }}$ est un antipode du monoïde quantique $(X, m, e)$, 
si le diagramme suivant d'applications $K$-linéaires est commutatif

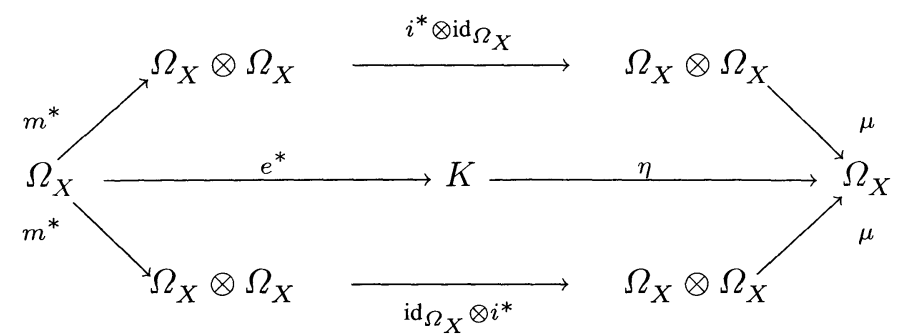

où $\mu$ désigne l'application $K$-linéaire définissant la multiplication et $\eta$ celle définissant l'unité de l'algèbre $\Omega_{X}$.

Le diagramme ci-dessus n'est pas un diagramme de morphismes d'algèbres et il ne peut donc pas être traduit en un diagramme de la catégorie des espaces quantiques. Ce diagramme exprime que $i^{*}$ est un antipode, au sens des bigèbres de Hopf ([Ab], p. 61), avec la seule différence qu'ici $\Omega_{X}$ est une bigèbre graduée gauche et en plus on impose à $i^{*}$ d'être compatible avec la différentielle. En particulier, on en déduit une structure de bigèbre de Hopf sur l'algèbre des observables $\mathscr{Q}_{X}$ [cf. (3.1.2)].

Théorème (4.1.2). i) Un monoïde quantique possède au plus un antipode.

ii) Si $(X, m, e)$ désigne un monoïde quantique et $i$ un antipode de ce monoïde, les diagrammes suivants sont commutatifs

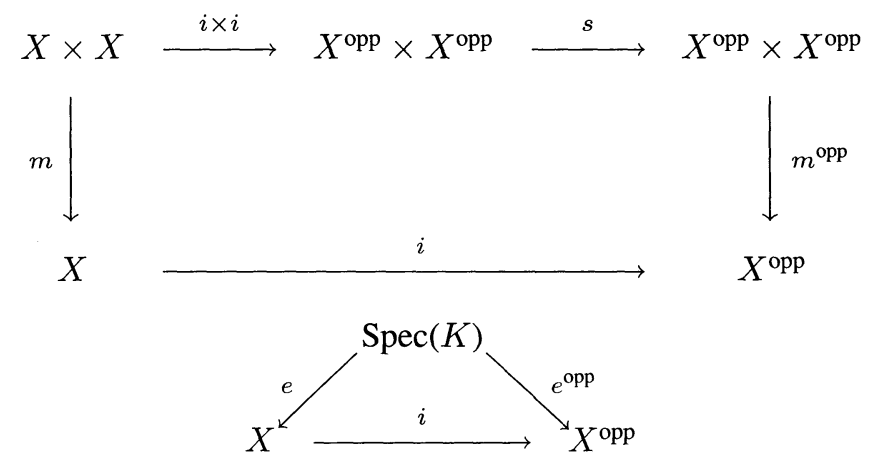

où s désigne la contrainte de commutativité [cf. (2.3.7)].

La démonstration est une transposition immédiate de [Ab], p.61-64.

Définition (4.1.3). On appelle groupe quantique un quadruplet $(X, m, e, i)$, où $(X, m, e)$ est un monoïde quantique et $i$ un antipode de ce monoïde. On dit que $(X, m, e)$ est le monoïde quantique sous-jacent à $(X, m, e, i)$. Si $(X, m, e, i)$ et $\left(X^{\prime}, m^{\prime}, e^{\prime}, i^{\prime}\right)$ sont des groupes quantiques, un morphisme de groupes quantiques de $(X, m, e, i)$ dans $\left(X^{\prime}, m^{\prime}, e^{\prime}, i^{\prime}\right)$ est un morphisme $f$ des monoïdes quantiques sous-jacents tel que le diagramme suivant soit commutatif

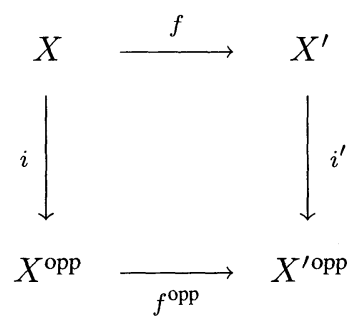


Exercise (4.1.4). Démontrer que la commutativité de ce diagramme résulte, en fait, de l'hypothèse que $f$ soit un morphisme de monoïdes quantiques.

\subsection{Groupe quantique associé à un monoïde quantique matriciel}

En généralisant une construction de Manin ([Man2], p.44, théorème 3), on peut associer à tout monoïde quantique matriciel un groupe quantique, correspondant en quelque sorte au "groupe des éléments inversibles" de ce monoïde. On obtient le théorème suivant dont la démonstration est identique à celle de Manin.

Théorème (4.2.1). Soit $(X, m, e)$ un monoïde quantique matriciel. Il existe un groupe quantique $(\tilde{X}, \tilde{m}, \tilde{e}, i)$ et un morphisme de monoïdes quantiques $f_{0} d u$ monoïde quantique sous-jacent à $(\tilde{X}, \tilde{m}, \tilde{e}, i)$ dans $(X, m, e)$, possédant la propriété universelle suivante: Pour tout groupe quantique $\left(X^{\prime}, m^{\prime}, e^{\prime}, i^{\prime}\right)$ et tout morphisme de monoides quantiques $f$ de $\left(X^{\prime}, m^{\prime}, e^{\prime}\right)$ dans $(X, m, e)$ il existe un morphisme unique de groupes quantiques $g$ de $\left(X^{\prime}, m^{\prime}, e^{\prime}, i^{\prime}\right)$ dans $(\tilde{X}, \tilde{m}, \tilde{e}, i)$ tel que $f=f_{0} \circ g$. De plus, l'algèbre $\Omega_{\tilde{X}}$ est engendrée par les images itérées de $f_{0}^{*}\left(\Omega_{X}\right)$ par $i^{*}$, autrement dit, toute sousalgèbre de $\Omega_{\tilde{X}}$ contenant $f_{0}^{*}\left(\Omega_{X}\right)$ et stable par $i^{*}$ est égale à $\Omega_{\tilde{X}}$.

(4.2.2). On dira que $(\tilde{X}, \tilde{m}, \tilde{e}, i)$ est le groupe quantique associé au monoïde quantique matriciel $(X, m, e)$ et $f_{0}$ le morphisme canonique. Si $(X, m, e)$ est le monoïde quantique des endomorphismes d'un cône quantique $C$ (3.4.3), on dira que $(\tilde{X}, \tilde{m}, \tilde{e}, i)$ est le groupe quantique des automorphismes de $C$.

(4.2.3). Le monoïde quantique sous-jacent au groupe quantique associé à un monoïde quantique matriciel n'est pas en général un monoïde quantique matriciel, ni même de type fini, ce qui enlève une grande partie de l'interêt de cette construction. Néanmoins on définira (4.3.1) une classe de monoïdes quantiques matriciels dont le groupe quantique associé est matriciel [prop. (4.3.3)].

(4.2.4). On peut également généralisier dans le cadre du présent travail les autres constructions de Manin ([Man2], p.46) pour obtenir un groupe quantique dont l'antipode est un isomorphisme ou même une involution.

\subsection{Monoïdes quantiques de Cramer}

Définition (4.3.1). On dit qu'un monoïde quantique $(X, m, e)$ est de Cramer à gauche (resp. à droite), s'il existe une matrice multiplicative $A$, dont les coefficients engendrent la $K$-algèbre différentielle $\Omega_{X}$, une matrice $B$ à coefficients dans $\mathscr{Q}_{X}$ et un élément $t$ de $\mathscr{Q}_{X}$, tels que

$$
m^{*}(t)=t \otimes t, \quad e^{*}(t)=1
$$

et

$$
B A=t I \quad(\text { resp. } A B=t I)
$$

(où $I$ désigne la matrice unité). On dit qu'un monoïde quantique est de Cramer, s'il est à la fois de Cramer à gauche et de Cramer à droite.

On remarque qu'un monoïde quantique de Cramer à gauche ou à droite est en particulier un monoïde quantique matriciel. 
Proposition (4.3.2). Pour que le monö̈de quantique sous-jacent à un groupe quantique soit de Cramer, il faut et il suffit qu'il soit matriciel.

Démonstration. Si $(X, m, e, i)$ désigne un groupe quantique et $A$ une matrice multiplicative, on a

$$
i^{*}(A) \cdot A=A \cdot i^{*}(A)=I
$$

[cf. (3.2.1) et (4.1.1)], ce qui démontre la proposition.

Proposition (4.3.3). Soient $(X, m, e)$ un monoïde quantique de Cramer à gauche (ou à droite) et $(\tilde{X}, \tilde{m}, \tilde{e}, i)$ le groupe quantique associé. Alors $(\tilde{X}, \tilde{m}, \tilde{e})$ est un monoïde quantique matriciel.

Démonstration. Soit $f_{0}: \tilde{X} \rightarrow X$ le morphisme canonique. Par hypothèse, il existe une matrice multiplicative $A$, dont les coefficients engendrent la $K$-algèbre différentielle $\Omega_{X}$, une matrice $B$ à coefficients dans $\mathscr{O}_{X}$ et un élément $t$ de $\mathscr{Q}_{x}$ tels que

$$
m^{*}(t)=t \otimes t, \quad e^{*}(t)=1
$$

et

$$
B A=t I \text {. }
$$

Posons $\tilde{t}=f_{0}^{*}(t)$ et $s=i^{*}(\tilde{t})$. La condition (4.3.3.1) implique que

$$
s \tilde{t}=\tilde{t} s=1
$$

autrement dit, que $\tilde{t}$ est inversible d'inverse $s$, d'où

$$
i^{*}(s)=\tilde{t} \text {. }
$$

La condition (4.3.3.2) implique, en vertu de (4.3.3.3), que si l'on pose

$$
\tilde{A}=f_{0}^{*}(A) \text { et } \tilde{B}=f_{0}^{*}(B) \text {, }
$$

on a

$$
s \tilde{B} \tilde{A}=I .
$$

Comme $A$ est une matrice multiplicative, il en est de même pour $\tilde{A}$, d'où

$$
i^{*}(\tilde{A}) \tilde{A}=\tilde{A} i^{*}(\tilde{A})=I,
$$

et les conditions (4.3.3.5) et (4.3.3.6) impliquent que

$$
i^{*}(\tilde{A})=s \tilde{B} \text {. }
$$

Démontrons que l'algèbre différentielle $\Omega_{\tilde{X}}$ est engendrée par les coefficients de $\tilde{A}$ et $s$. Pour cela, en vertu de (4.2.1), il suffit de démontrer que la sous-algèbre différentielle $\Omega$ de $\Omega_{\tilde{X}}$ engendrée par les coefficients de $\tilde{A}$ et $s$ (qui contient $f_{0}^{*}\left(\Omega_{X}\right)$, puisque $\Omega_{X}$ est engendrée par les coefficients de $A$ ) est stable par $i^{*}$. Comme $i^{*}$ commute à la différentielle et inverse l'ordre des facteurs d'un produit, il suffit simplement de voir que les coefficients de $i^{*}(\tilde{A})$ et $i^{*}(s)$ sont dans $\Omega$. Mais cela résulte de (4.3.3.4) et (4.3.3.7) et du fait que $\Omega$ contient $f_{0}^{*}\left(\Omega_{X}\right)$. Pour terminer la démonstration, il suffit de remarquer que

$$
\left(\begin{array}{cc}
\tilde{A} & 0 \\
0 & s
\end{array}\right)
$$

est une matrice multiplicative à coefficients dans $\mathscr{\odot}_{\tilde{X}}$. 
Exemple (4.3.4). On démontre que le monoïde quantique défini par les relations (1) à (18) du théorème (3.5.5) est un monoïde quantique de Cramer. Explicitons le cas où $n$ est égal à 2 . Ce monoïde est alors isomorphe [Mal1] à

$$
(X, m, e)=(\operatorname{Spec}(\Omega), \operatorname{Spec}(\Delta), \operatorname{Spec}(\varepsilon)),
$$

où $\Omega=K\langle\langle a, b, c, d\rangle\rangle / J, a, b, c$ et $d$ désignent des indéterminées, $J$ est l'idéal bilatère de $K\langle\langle a, b, c, d\rangle\rangle$ engendré par

$$
\begin{gathered}
b a-p a b, d c-p c d, c a-q a c, d b-q b d, p c b-q b c, \\
q(d a-a d)-q^{2} b c+c b\left(\Leftrightarrow p(d a-a d)-p^{2} c b+b c\right), \\
\alpha a-p q a \alpha, \beta b-p q b \beta, \gamma c-p q c \gamma, \delta d-p q d \delta, \\
\alpha c-p c \alpha, \beta d-p d \beta, \alpha b-q b \alpha, \gamma d-q d \gamma, \\
\beta a-p a \beta-(p q-1) b \alpha, \delta c-p c \delta-(p q-1) d \gamma, \\
\gamma a-q a \gamma-(p q-1) c \alpha, \delta b-q b \delta-(p q-1) d \beta, \\
\alpha d-d \alpha, q \beta c-p c \beta-(p q-1) d \alpha, p \gamma b-q b \gamma-(p q-1) d \alpha, \\
p q \delta a-p q a \delta-q(p q-1) b \gamma-p(p q-1) c \beta-(p q-1)^{2} d \alpha, \\
\alpha^{2}, \beta^{2}, \gamma^{2}, \delta^{2}, \\
\alpha \gamma+p \gamma \alpha, \beta \delta+p \delta \beta, \alpha \beta+q \beta \alpha, \gamma \delta+q \delta \gamma, \\
\delta \alpha+\alpha \delta, \quad p \gamma \beta+q \beta \gamma-(p q-1) \alpha \delta .
\end{gathered}
$$

(où $p, q$ désignent des éléments inversibles de $K$ et $\alpha, \beta, \gamma$ et $\delta$ les différentielles de $a$, $b, c$ et $d$ respectivement) et $\Delta$ (resp. $\varepsilon$ ) l'unique morphisme d'algèbres différentielles tel que

$$
\begin{gathered}
\Delta(a)=a \otimes a+b \otimes c, \\
\Delta(b)=a \otimes b+b \otimes d, \\
\Delta(c)=c \otimes a+d \otimes c, \\
\Delta(d)=c \otimes b+d \otimes d, \\
\text { (resp. } \varepsilon(a)=\varepsilon(d)=1 \text { et } \varepsilon(b)=\varepsilon(c)=0) .
\end{gathered}
$$

Alors la matrice

$$
A=\left(\begin{array}{ll}
a & b \\
c & d
\end{array}\right)
$$

est une matrice multiplicative dont les coefficients engendrent la $K$-algèbre différentielle $\Omega$, et si l'on pose

$$
t=a d-q^{-1} c b=d a-q b c=d a-p c b=a d-p^{-1} b c,
$$

on a

$$
\Delta(t)=t \otimes t, \quad \varepsilon(t)=1
$$

et

$$
\left(\begin{array}{cc}
d & -q b \\
-q^{-1} c & a
\end{array}\right)\left(\begin{array}{ll}
a & b \\
c & d
\end{array}\right)=\left(\begin{array}{ll}
a & b \\
c & d
\end{array}\right)\left(\begin{array}{cc}
d & -p b \\
-p^{-1} c & a
\end{array}\right)=t I,
$$

ce qui prouve que le monoïde quantique $(X, m, e)$ est un monoïde quantique de Cramer. De plus, on démontre que si $(\tilde{X}, \tilde{m}, \tilde{e}, i)$ désigne le groupe quantique associé au monoïde quantique $(X, m, e)$, alors $\Omega_{\tilde{X}}=K\langle\langle a, b, c, d, s\rangle\rangle / J^{\prime}$, où $s$ désigne une nouvelle indéterminée et $J^{\prime}$ est l'idéal différentiel de $K\langle\langle a, b, c, d, s\rangle\rangle$ engendré par 
$J$, st -1 et $t s-1$, autrement dit, $\Omega_{\tilde{X}}$ s'obtient de $\Omega_{X}$ en "inversant" l'élément $t$, qui s'appelle le déterminant quantique.

\section{Références}

[Ab] Abe, E.: Hopf algebras. Cambridge Tracts in Math. 74, Cambridge: Cambridge Univ. Press, 1980

[Ao1] Aomoto, K.: $q$-analogue of the de Rham cohomology associated with Jackson integrals. I. Proc. Jap. Acad. A 66, 161-164 (1990)

[Ao2] Aomoto, K.: $q$-analogue of the de Rham cohomology associated with Jackson integrals. II. Proc. Jap. Acad. A 66, 240-244 (1990)

[Ao3] Aomoto, K.: Finiteness of a cohomology associated with certain Jackson integrals. Tôhoku Math. J. 43, 75-101 (1991)

[AST] Artin, M., Schelter, W., Tate, J.: Quantum deformations of $G L_{n}$. Commun. Pure Appl. Math. 44, 879-895 (1991)

[Be] Bergman, G.M.: The diamond lemma for ring theory. Adv. Math. 29, 178-218 (1978)

[Bd] Bernard, D.: Quantum Lie algebras and differential calculus on quantum groups. Preprint 1990

[Bo] Bourbaki, N.: Élements de Mathématique. Algèbre, chapitres 1 à 3, Paris: Hermann 1970

[Br] Brzeziński, T.: Exterior bialgebras. Preprint 1991

[BDR] Brzeziński, T., Dabrowsky, H., Rembieliński, J.: On the quantum differential calculus and the quantum holomorphicity. J. Math. Phys. 33, 1, 19-24 (1992)

[CSWW] Carow-Watamura, U., Schlieker, M., Watamura, S., Weich, W.: Bicovariant differential calculus on quantum groups $S U_{q}(N)$ and $S O_{q}(N)$. Commun. Math. Phys. 142, 605-641 (1991)

[Ca] Cartier, P.: Calcul différentiel non commutatif, Exposés à l'E.N.S. (1989)

[Co] Connes, A.: Non-commutative differential geometry. Publications Mathématiques de l'I.H.E.S. 62, 41-144 (1985)

[DM] Deligne, P., Milne, J.S.: Tannakian categories. In: Hodge cycles, motives, and Shimura varieties. Lect. Notes in Math. 900, Berlin, Heidelberg, New York: Springer 1982, pp. 101-228

[Dr] Drinfel'd, V.G.: Quantum groups. In: Proceedings of the International Congress of Mathematicians 1986, Berkeley, Providence, RI: AMS, 1987, pp. 798-820

[DV1] Dubois-Violette, M.: Dérivations et calcul différentiel non commutatif. C.R.A.S. 307, 403-408 (1988)

[DV2] Dubois-Violette, M.: Noncommutative differential geometry, quantum mechanics and gauge theory. Preprint, L.P.T.H.E.-Orsay 90/31 (1990)

[DKM1] Dubois-Violette, M., Kerner, R., Madore, J.: Noncommutative differential geometry of matrix algebras. J. Math. Phys. 31, 2, 316-322 (1990)

[DKM2] Dubois-Violette, M., Kerner, R., Madore, J.: Noncommutative differential geometry and new models of gauge theory. J. Math. Phys. 31, 2, 323-330 (1990)

[FT] Feng, P., Tsygan, B.: Hochschild and cyclic homology of quantum groups. Commun. Math. Phys. 140, 481-521 (1991)

[EGA IV $\mathrm{I}_{4}$ Grothendieck, A.: Éléments de géométrie algébrique IV. Publications Mathématiques de l'I.H.E.S. 32, (1967)

[Gr] Grothendieck, A.: On the de Rham cohomology of algebraic varieties. Publications Mathématiques de l'I.H.E.S. 29, 95-103 (1966)

[GRR] Gurevich, D., Radul, A., Rubtsov, V.: Non-commutative differential geometry and YangBaxter equation. Preprint 1990

[HW] Hibi, T., Wakayama, M.: A $q$-analogue of Capelli's identity for $G L(2)$. Preprint, Hokkaido University 1991

[J1] Jimbo, M.: A $q$-difference analogue of $U(g)$ and the Yang-Baxter equation. Lett. Math. Phys. 10, 63-69 (1985)

[J2] Jimbo, M.: Quantum $R$ matrix for the generalized Toda system. Commun. Math. Phys. 102, 537-547 (1986)

[J3] Jimbo, M.: A $q$-analogue of $U(g l(N+1))$, Hecke algebras and the Yang-Baxter equation. Lett. Math. Phys. 11, 247-252 (1986) 
[Ju] Jurčo, B.: Differential calculus on quantized simple Lie groups. Lett. Math. Phys. 22, 177-186 (1991)

[Ka] Karoubi, M.: Homologie cyclique et $K$-théorie. Asterisque 149, (1987)

[Kas] Kassel, C.: Cyclic homology of differential operators, the Virasoro algebra and a $q$ analogue. Preprint 1991

[Mal1] Maltsiniotis, G.: Groupes quantiques et structures différentielles. C.R.A.S. 311, Sér. I, 831-834 (1990)

[Ma12] Maltsiniotis, G.: Calcul différentiel sur le groupe linéaire quantique. Preprint 1990

[Mal3] Maltsiniotis, G.: Groupoïdes quantiques. C.R.A.S. 314, Sér. I, 249-252 (1992)

[Man1] Manin, Yu.I.: Some remarks on Koszul algebras and quantum groups. Ann. Inst. Fourier 37, 4, 191-205 (1987)

[Man2] Manin, Yu.I.: Quantum groups and non-commutative geometry. Centre de Recherches Mathématiques de l'Université de Montréal 1988

[Man3] Manin, Yu.I.: Multiparametric quantum deformation of the general linear supergroup. Commun. Math. Phys. 123, 163-175 (1989)

[Man4] Manin, Yu.I.: Notes on quantum groups and quantum de Rham complexes. Preprint, MPI/91-60 (1991)

[MNW1] Masuda, T., Nakagami, Y., Watanabe, J.: Noncommutative differential geometry on the quantum $S U(2)$, I: An algebraic viewpoint. $K$-Theory 4, 157-180 (1990)

[MNW2] Masuda, T., Nakagami, Y., Watanabe, J.: Noncommutative differential geometry on the quantum two sphere of Podles. I: An algebraic viewpoint. $K$-Theory 5, 151-175 (1991)

[M-H] Müller-Hoissen, F.: Differential calculi on the quantum group $G L_{p, q}(2)$. Preprint, GOET-TP 55/91 (1991)

[NUW] Noumi, M., Umeda, T., Wakayama, M.: A quantum analogue of the Capelli identity and an elementary differential calculus on $G L_{q}(n)$. Preprint, University of Toyko 1991

[Po] Podles, P.: Differential calculus on quantum spheres. Lett. Math. Phys. 18, 107-119 (1989)

[RTF] Reshetikhin, N.Yu., Takhtadzhyan, L.A., Fadeev, L.D.: Quantization of Lie groups and Lie algebras. Len. Math. J. 1, 1, 193-225 (1990)

[Ro] Rosso, M.: Algèbres enveloppantes quantifiées, groupes quantiques compacts de matrices et calcul différentiel non commutatif. Duke Math. J. 61, 1, 11-40 (1990)

[Sch] Schirrmacher, A.: Remarks on the use of $R$-matrices. Preprint 1991

[SWZ] Schirrmacher, A., Wess, J., Zumino, B.: The two-parameter deformation of $G L(2)$, its differential calculus, and Lie algebra. Z. Phys. C - Particles and Fields 49, 317-324 (1991)

[SVZ] Schmidke, W.B., Vokos, S.P., Zumino, B.: Differential geometry of the quantum supergroup $G L_{q}(1 \mid 1)$. Z. Phys. C - Particles and Fields 48, 249-255 (1990)

[Su] Sudbery, A.: Consistent multiparameter quantisation of $G L(n)$. J. Phys. A: Math. Gen. 23, L 697-L 704 (1990)

[Ta] Takhtadzhyan, L.A.: Noncommutative homology of quantum tori. Funct. Anal. Appl. 23, 2, 147-149 (1989)

[Tsy] Tsygan, B.: Notes on differential forms on quantum groups. Preprint 1991

[WZ] Wess, J., Zumino, B.: Covariant differential calculus on the quantum hyperplane. Preprint 1990

[Wo1] Woronowicz, S.L.: Twisted $S U(2)$ group. An example of a non-commutative differential calculus. Publ. RIMS 23, 117-181 (1987)

[Wo2] Woronowicz, S.L.: Differential calculus on compact matrix pseudogroups (quantum groups). Commun. Math. Phys. 122, 125-170 (1989)

[Za1] Zakrzewski, S.: A differential structure for quantum mechanics. J. Geom. Phys. 2, 3, 135-145 (1985)

[Za2] Zakrzewski, S.: Quantum and classical pseudogroups. Part. II. Differential and symplectic pseudogroups. Commun. Math. Phys. 134, 371-395 (1990) 